\title{
Gold(I)-catalyzed Oxidation of Acyl Acetylenes to Vicinal Tricarbonyls
}

\author{
Alexey Yu. Dubovtsev,* Dmitry V. Dar'in, Vadim Yu. Kukushkin* \\ Saint Petersburg State University, Universitetskaya Nab. 7/9 199034 Saint Petersburg, Russian Federation \\ E-mail: a.dubovtsev@spbu.ru (A.Yu.D.), v.kukushkin@spbu.ru (V.Yu.K.)
}

\section{Table of Contents}

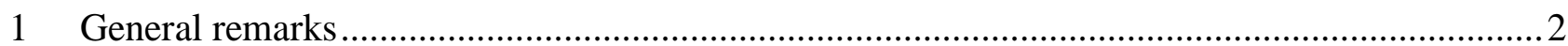









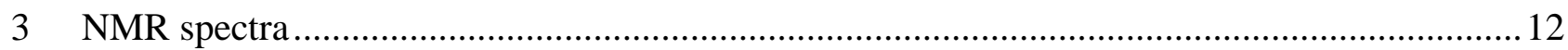




\section{General remarks}

NMR spectra were recorded at ambient temperature with a Bruker Avance III 400 instrument at $400.13 \mathrm{MHz}\left({ }^{1} \mathrm{H} \mathrm{NMR}\right)$ and $100.61 \mathrm{MHz}\left({ }^{13} \mathrm{C} \mathrm{NMR}\right)$ in $\mathrm{CDCl}_{3}$ or DMSO- $d_{6}$. Chemical shifts $(\delta)$ are given in ppm relative to resonances of the solvents $\left({ }^{1} \mathrm{H}: \delta=7.26\right.$ for residual $\mathrm{CHCl}_{3}$ peak, $\delta=2.50$ for residual DMSO peak; ${ }^{13} \mathrm{C}: \delta=77.2$ for $\mathrm{CDCl}_{3}, \delta=39.5$ for DMSO- $d_{6}$ ). Mass-spectra were recorded on Bruker MicroTOF (ESI) and Bruker maXis HRMS-ESI-QTOF instruments. Chromatographic separation was carried out on Macherey-Nagel silica gel $60 \mathrm{M}(0.04-0.063 \mathrm{~mm})$. Analytical TLC was performed on unmodified Merck ready-to-use plates (TLC silica gel 60 F254); detection was achieved with a UV lamp. Melting points were measured with Stuart smp30 apparatus. $\mathrm{Ph}_{3} \mathrm{PAuNTf}_{2}{ }^{1}$ and $N$-oxides $\mathbf{3 a - \mathbf { d } ^ { 2 , 3 }}$ were synthesized accordingly to the published protocols. Alkynyl esters, ${ }^{4}$ ketones $^{5}$ and amides ${ }^{6}$ were prepared by the literature procedures. The solvents and other reagents were purchased from commercial vendors and were used as received.

\section{Experimental procedures}
<smiles>[R]C(=O)C([R])=O</smiles>


\subsection{General procedure for the synthesis of tricarbonyl compounds $\mathbf{5}$}

$\mathrm{Ph}_{3} \mathrm{PAuNTf}_{2}(7.4 \mathrm{mg}, 10.0 \mu \mathrm{mol}, 5 \mathrm{~mol} \%)$ was added to the solution of alkyne $1(0.2 \mathrm{mmol})$ and 2,6dichloropyridine $N$-oxide 3d $(82.0 \mathrm{mg}, 0.5 \mathrm{mmol}, 2.5$ equiv) in DCM $(0.4 \mathrm{~mL})$. The resulting mixture was stirred at room temperature for $1 \mathrm{~h}$. The solvent was then removed in vacuo and the residue was purified by silica gel chromatography eluting with hexane/EtOAc (gradient from $4: 1$ to $1: 1)$ to afford tricarbonyl compounds $\mathbf{5}$ or its hydrates $\mathbf{5}$ '.

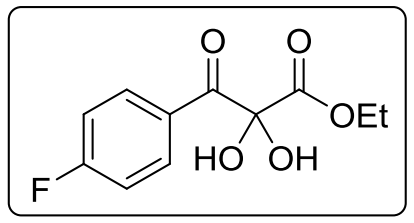

Ethyl 3-(4-fluorophenyl)-2,2-dihydroxy-3-oxopropanoate (5'a): yellowish solid (40.7 mg, 84\%); mp 75.0-76.0 ${ }^{\circ} \mathrm{C}$ (hexane/ethyl acetate); $\mathrm{R}_{f} 0.35$ (hexane/ethyl acetate 2:1); ${ }^{1} \mathbf{H}$ NMR (400 MHz, DMSO- $\left.d_{6}\right) \delta 8.14-8.09(\mathrm{~m}, 2 \mathrm{H}, \mathrm{Ar}), 7.59$ (br.s, $\left.1 \mathrm{H}, \mathrm{OH}\right), 7.35$ (t, $J=$ $8.9 \mathrm{~Hz}, 2 \mathrm{H}, \mathrm{Ar}), 4.09$ (q, $\left.J=7.1 \mathrm{~Hz}, 2 \mathrm{H}, \mathrm{CH}_{2}\right), 1.04(\mathrm{t}, J=7.1 \mathrm{~Hz}, 3 \mathrm{H}, \mathrm{Me}) ;{ }^{13} \mathbf{C} \mathbf{N M R}(100$ 
MHz, DMSO- $\left.d_{6}\right) \delta 192.1,169.6,165.1\left(\mathrm{~d}, J_{F}=252.8 \mathrm{~Hz}, \mathrm{CF}\right), 132.7\left(\mathrm{~d}, J_{F}=9.5 \mathrm{~Hz}, \mathrm{CH}\right)$, $129.6\left(\mathrm{~d}, J_{F}=2.8 \mathrm{~Hz}, \mathrm{C}\right), 115.6\left(\mathrm{~d}, J_{F}=22.0 \mathrm{~Hz}, \mathrm{CH}\right), 94.2,61.2,13.7$; HRMS (ESI) for keto form: $m / z[\mathrm{M}+\mathrm{Na}]^{+}$calcd. for $\mathrm{C}_{11} \mathrm{H}_{9} \mathrm{FNaO}_{4}{ }^{+}: 247.0383$; found: 247.0385 .

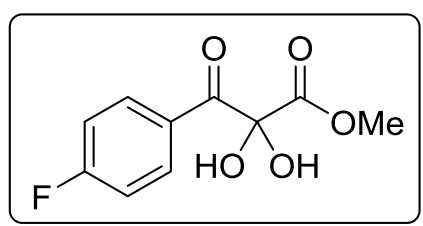

Methyl 3-(4-fluorophenyl)-2,2-dihydroxy-3-oxopropanoate $\left(\mathbf{5}^{\prime} \mathbf{b}\right)^{7}$ : yellowish solid $(38.8 \mathrm{mg}, \quad 85 \%)$; $\mathrm{mp} \quad 68.5-69.5{ }^{\circ} \mathrm{C}$ (hexane/ethyl acetate); $\mathrm{R}_{f} 0.35$ (hexane/ethyl acetate 2:1); ${ }^{1} \mathbf{H}$ NMR (400 MHz, DMSO- $\left.d_{6}\right) \delta 8.14-8.11$ (m, 2H, Ar), 7.64 (br.s, 2H, OH), $7.35(\mathrm{t}, J=8.9 \mathrm{~Hz}, 2 \mathrm{H}, \mathrm{Ar}), 3.64$ (s, 3H, Me); ${ }^{13} \mathrm{C}$ NMR (100 MHz, DMSO- $\left.d_{6}\right) \delta 192.1,170.2$, $165.1\left(\mathrm{~d}, J_{F}=252.8 \mathrm{~Hz}, \mathrm{CF}\right), 132.7\left(\mathrm{~d}, J_{F}=9.6 \mathrm{~Hz}, \mathrm{CH}\right), 129.5\left(\mathrm{~d}, J_{F}=2.9 \mathrm{~Hz}, \mathrm{C}\right), 115.7(\mathrm{~d}$, $\left.J_{F}=22.0 \mathrm{~Hz}, \mathrm{CH}\right), 94.5,52.4$; HRMS (ESI) for keto form: $m / z[\mathrm{M}+\mathrm{Na}]^{+}$calcd. for $\mathrm{C}_{10} \mathrm{H}_{7} \mathrm{FNaO}_{4}^{+}$: 233.0226; found: 233.0217 .



Ethyl 2,2-dihydroxy-3-oxo-3-phenylpropanoate $\left(5^{\prime} c\right)^{7}$ : yellowish oil (39.0 mg, 87\%); $\mathrm{R}_{f} 0.40$ (hexane/ethyl acetate 2:1); ${ }^{\mathbf{1}} \mathbf{H}$ NMR (400 MHz, DMSO- $\left.d_{6}\right) \delta 8.03(\mathrm{~d}, J=7.0 \mathrm{~Hz}, 2 \mathrm{H}, \mathrm{Ph}), 7.62(\mathrm{t}, J=7.4 \mathrm{~Hz}, 1 \mathrm{H}, \mathrm{Ph})$, 7.53-7.49 (m, 3H, Ph+OH), $4.08\left(\mathrm{q}, J=7.1 \mathrm{~Hz}, 2 \mathrm{H}, \mathrm{CH}_{2}\right), 1.01(\mathrm{t}, J=7.1 \mathrm{~Hz}, 3 \mathrm{H}, \mathrm{Me}) ;{ }^{13} \mathrm{C}$ NMR (100 MHz, DMSO-d6) $\delta$ 193.4, 169.6, 133.5, 132.9, 129.5, 128.5, 94.0, 61.1, 13.7; HRMS (ESI) for keto form: $m / z[\mathrm{M}+\mathrm{Na}]^{+}$calcd. for $\mathrm{C}_{11} \mathrm{H}_{10} \mathrm{NaO}_{4}{ }^{+}$: 229.0477; found: 229.0482 .



Methyl 2,2-dihydroxy-3-oxo-3-phenylpropanoate $\left(5^{\prime} \mathbf{d}\right)^{7}$ : yellowish oil (38.7 mg, 92\%); $\mathrm{R}_{f} 0.40$ (hexane/ethyl acetate 2:1); ${ }^{1} \mathbf{H}$ NMR (400 MHz, DMSO-d6) $\delta 8.04-8.02(\mathrm{~m}, 2 \mathrm{H}, \mathrm{Ph}), 7.63(\mathrm{t}, J=7.4 \mathrm{~Hz}, 1 \mathrm{H}, \mathrm{Ph}), 7.59$ (br.s, $1 \mathrm{H}, \mathrm{OH}), 7.51(\mathrm{t}, J=7.7 \mathrm{~Hz}, 2 \mathrm{H}, \mathrm{Ph}), 3.62$ (s, 3H, Me); ${ }^{13} \mathrm{C}$ NMR (100 MHz, DMSO- $\left.d_{6}\right) \delta$ 193.4, 170.2, 133.6, 132.8, 129.6, 128.5, 94.2, 52.4; HRMS (ESI) for hydrate form: $m / z$ [M + $\mathrm{Na}]^{+}$calcd. for $\mathrm{C}_{10} \mathrm{H}_{10} \mathrm{NaO}_{5}^{+}$: 233.0426; found: 233.0424 .

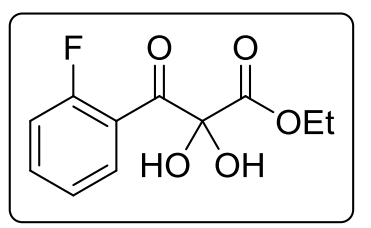

Ethyl 3-(2-fluorophenyl)-2,2-dihydroxy-3-oxopropanoate (5'e): yellowish oil (34.9 mg, 72\%); $\mathrm{R}_{f} 0.35$ (hexane/ethyl acetate 2:1); ${ }^{1} \mathbf{H}$ NMR (400 MHz, DMSO- $\left.d_{6}\right) \delta$ 7.94-7.89 (m, 1H, Ar), 7.67-7.61 (m, 1H, Ar), 7.51 (br.s, 2H, OH), 7.33-7.27 (m, 2H, Ar), 4.11 (q, $J=7.1$ $\left.\mathrm{Hz}, 2 \mathrm{H}, \mathrm{CH}_{2}\right), 1.08$ (t, $\left.J=7.1 \mathrm{~Hz}, 3 \mathrm{H}, \mathrm{Me}\right) ;{ }^{13} \mathrm{C}$ NMR (100 MHz, DMSO-d $) \delta 193.0,168.9$, $160.4\left(\mathrm{~d}, J_{F}=255.7 \mathrm{~Hz}, \mathrm{CF}\right), 134.8\left(\mathrm{~d}, J_{F}=9.3 \mathrm{~Hz}, \mathrm{CH}\right), 131.0\left(\mathrm{~d}, J_{F}=2.2 \mathrm{~Hz}, \mathrm{CH}\right), 124.3$ $\left(\mathrm{d}, J_{F}=3.6 \mathrm{~Hz}, \mathrm{CH}\right), 122.8\left(\mathrm{~d}, J_{F}=11.6 \mathrm{~Hz}, \mathrm{C}\right), 116.6\left(\mathrm{~d}, J_{F}=22.4 \mathrm{~Hz}, \mathrm{CH}\right), 94.5,61.0,13.7$; HRMS (ESI) for keto form: $m / z[\mathrm{M}+\mathrm{Na}]^{+}$calcd. for $\mathrm{C}_{11} \mathrm{H}_{9} \mathrm{FNaO}_{4}{ }^{+}$: 247.0383; found: 247.0381 . 


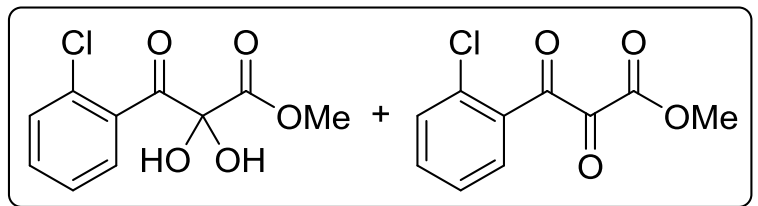

Methyl 3-(2-chlorophenyl)-2,3-dioxopropanoate (5'f) and methyl 3-(2-chlorophenyl)-2,2dihydroxy-3-oxopropanoate (5f): yellowish oil (32.0 mg, 67\%); $\mathrm{R}_{f} 0.30$ (hexane/ethyl acetate 2:1); hydrate form: keto form $=2: 1 ;{ }^{1} \mathbf{H}$ NMR for hydrate form (400 MHz, DMSO- $\left.d_{6}\right) \delta 7.82$ $(\mathrm{dd}, J=7.5,1.4 \mathrm{~Hz}, 1 \mathrm{H}, \mathrm{Ar}), 7.65-7.48(\mathrm{~m}, 4 \mathrm{H}, \mathrm{Ar}+2 \mathrm{OH}), 7.41(\mathrm{td}, J=7.4,7.0,1.8 \mathrm{~Hz}, 1 \mathrm{H}$, Ar), 3.66 (s, 3H, OMe); ${ }^{1} \mathbf{H}$ NMR for keto form (400 MHz, DMSO- $d_{6}$ ) $\delta 7.99$ (dd, $J=7.8,1.8$ $\mathrm{Hz}, 1 \mathrm{H}, \mathrm{Ar}), 7.82-7.78(\mathrm{~m}, 1 \mathrm{H}, \mathrm{Ar}), 7.71$ (t, $J=7.3 \mathrm{~Hz}, 1 \mathrm{H}, \mathrm{Ar}), 7.65-7.48$ (m, 1H, Ar), 3.93 (s, 3H, OMe); ${ }^{13} \mathbf{C}$ NMR for hydrate and keto form (100 MHz, DMSO-d6) $\delta$ 196.3, 190.9, $181.7,169.7,158.9,136.5,134.8,133.7,133.2,132.2,131.1,130.8,130.4,129.8,129.6$, 128.3, 126.5, 95.0, 53.8, 52.3; HRMS (ESI) for keto form: $\mathrm{m} / z[\mathrm{M}+\mathrm{Na}]^{+}$calcd. for $\mathrm{C}_{10} \mathrm{H}_{7} \mathrm{ClNaO}_{4}^{+}$: 248.9931; found: 248.9936 .



\section{Ethyl 2,2-dihydroxy-3-oxo-3-(4-tolyl)propanoate}

$\left(5^{\prime} \mathbf{g}\right)^{7}:$ yellowish oil (41.0 mg, 86\%); $\mathrm{R}_{f} 0.35$ (hexane/ethyl acetate 2:1); ${ }^{1}$ H NMR $\left(400 \mathrm{MHz}, \mathrm{DMSO}-d_{6}\right) \delta 7.93(\mathrm{~d}, J=8.3 \mathrm{~Hz}, 2 \mathrm{H}, \mathrm{Ar}), 7.46$ (br.s, 2H, OH), $7.31(\mathrm{~d}, J=8.1 \mathrm{~Hz}, 2 \mathrm{H}, \mathrm{Ar}), 4.08(\mathrm{q}, J=7.1 \mathrm{~Hz}$, $\left.2 \mathrm{H}, \mathrm{CH}_{2}\right), 2.36(\mathrm{~s}, 3 \mathrm{H}, \mathrm{Me}), 1.02(\mathrm{t}, J=7.1 \mathrm{~Hz}, 3 \mathrm{H}, \mathrm{Me}) ;{ }^{13} \mathrm{C}$ NMR $\left(100 \mathrm{MHz}, \mathrm{DMSO}-d_{6}\right) \delta$ 192.9, 169.7, 144.0, 130.3 129.7, 129.0, 93.9, 61.0, 21.2, 13.7; HRMS (ESI) for keto form: $m / z[\mathrm{M}+\mathrm{Na}]^{+}$calcd. for $\mathrm{C}_{12} \mathrm{H}_{12} \mathrm{NaO}_{4}^{+}: 243.0633$; found: 243.0628 .

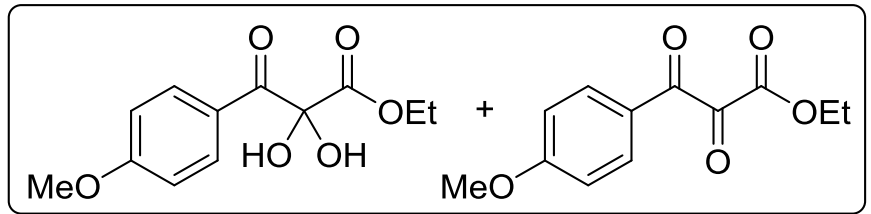

Ethyl 2,2-dihydroxy-3-(4-methoxyphenyl)-3-oxopropanoate (5'h) and ethyl 3-(4-methoxyphenyl)-2,3-dioxopropanoate $(5 \mathbf{h})^{7}$ : yellowish oil $(45.3 \mathrm{mg}, 91 \%)$; $\mathrm{R}_{f} 0.45$ (hexane/ethyl acetate 1:1); hydrate form:keto form $=7: 3 ;{ }^{1} \mathbf{H} \mathbf{~ N M R}$ for hydrate form $\left(400 \mathrm{MHz}, \mathrm{CDCl}_{3}\right) \delta 8.06(\mathrm{~d}, J=9.0 \mathrm{~Hz}, 2 \mathrm{H}, \mathrm{Ar}), 6.92$ (d, $J=9.0 \mathrm{~Hz}, 2 \mathrm{H}, \mathrm{Ar}$ ), 5.44 (br.s, 2H, $\mathrm{OH}), 4.20\left(\mathrm{q}, J=7.2 \mathrm{~Hz}, 2 \mathrm{H}, \mathrm{CH}_{2}\right), 3.86(\mathrm{~s}, 3 \mathrm{H}, \mathrm{OMe}), 1.10(\mathrm{t}, J=7.1 \mathrm{~Hz}, 3 \mathrm{H}, \mathrm{Me}) ;{ }^{13} \mathrm{C}$ NMR for hydrate form $\left(100 \mathrm{MHz}, \mathrm{CDCl}_{3}\right) \delta 190.0,170.3,164.8,132.9,124.3,114.2,91.7$, 63.2, 55.7, 13.8; ${ }^{1} \mathbf{H}$ NMR for keto form (400 MHz, $\left.\mathrm{CDCl}_{3}\right) \delta 7.98(\mathrm{~d}, J=8.9 \mathrm{~Hz}, 2 \mathrm{H}, \mathrm{Ar})$, $6.99(\mathrm{~d}, J=8.9 \mathrm{~Hz}, 2 \mathrm{H}, \mathrm{Ar}), 4.40\left(\mathrm{q}, J=7.2 \mathrm{~Hz}, 2 \mathrm{H}, \mathrm{CH}_{2}\right), 3.89$ (s, 3H, OMe), 1.37 (t, $J=7.1$ $\mathrm{Hz}, 3 \mathrm{H}, \mathrm{Me}) ;{ }^{13} \mathrm{C}$ NMR for keto form $\left(100 \mathrm{MHz}, \mathrm{CDCl}_{3}\right) \delta 188.4,184.1,165.7,161.0,132.8$, 124.7, 114.7, 63.3, 55.8, 14.1; HRMS (ESI) for keto form: $m / z[\mathrm{M}+\mathrm{Na}]^{+}$calcd. for $\mathrm{C}_{12} \mathrm{H}_{12} \mathrm{NaO}_{5}^{+}$: 259.0582; found: 259.0577 . 


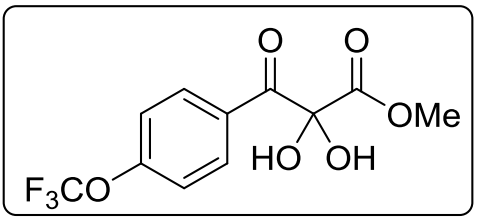

Methyl 2,2-dihydroxy-3-oxo-3-(4-(trifluoromethoxy)phenyl)propanoate (5'i): yellowish oil (44.1 $\mathrm{mg}, 75 \%) ; \mathrm{R}_{f}$ 0.45(hexane/ethyl acetate 1:1); ${ }^{\mathbf{1}} \mathbf{H}$ NMR (400 MHz, DMSO-d6) $\delta 8.16(\mathrm{~d}, J=8.9 \mathrm{~Hz}, 2 \mathrm{H}, \mathrm{Ar}), 7.72$ (br.s, $2 \mathrm{H}, \mathrm{OH}), 7.51(\mathrm{~d}, J=$ $8.5 \mathrm{~Hz}, 2 \mathrm{H}, \mathrm{Ar}), 3.65$ (s, 3H, Me); ${ }^{13} \mathrm{C}$ NMR (100 MHz, DMSO- $\left.d_{6}\right) \delta 192.4,170.1,151.6$, 133.2, 131.6, 120.5, 119.9 (q, $J_{F}=258.4 \mathrm{~Hz}, \mathrm{CF}_{3}$ ), 94.5, 52.4; HRMS (ESI) for keto form: $m / z[\mathrm{M}+\mathrm{Na}]^{+}$calcd. for $\mathrm{C}_{11} \mathrm{H}_{7} \mathrm{~F}_{3} \mathrm{NaO}_{5}{ }^{+}: 299.0143$; found: 299.0145 .

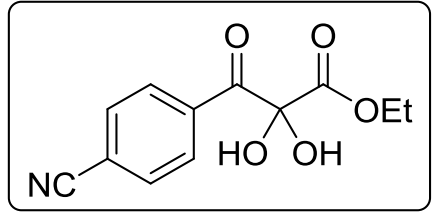

Ethyl 3-(4-cyanophenyl)-2,2-dihydroxy-3-oxopropanoate (5'j) ${ }^{8}$ : yellowish solid (41.4 $\mathrm{mg}, 83 \%)$; mp $104.0-105.5^{\circ} \mathrm{C}$ (hexane/ethyl acetate); $\mathrm{R}_{f} 0.40$ (hexane/ethyl acetate $\left.1: 1\right) ;{ }^{1} \mathbf{H}$ NMR (400 MHz, DMSO-d $d_{6} \delta 8.15(\mathrm{~d}, J=8.3 \mathrm{~Hz}, 2 \mathrm{H}, \mathrm{Ar}), 8.00(\mathrm{~d}, J=8.2 \mathrm{~Hz}, 2 \mathrm{H}$, Ar), 7.78 (br.s, 2H, OH), 4.11 (q, $\left.J=7.1 \mathrm{~Hz}, 2 \mathrm{H}, \mathrm{CH}_{2}\right), 1.05(\mathrm{t}, J=7.1 \mathrm{~Hz}, 3 \mathrm{H}, \mathrm{Me}){ }^{13} \mathbf{C} \mathbf{N M R}$ $\left(100 \mathrm{MHz}, \mathrm{DMSO}-d_{6}\right) \delta 193.2,169.3,136.5,132.6,130.1,118.1,115.4,94.4,61.4,13.7$; HRMS (ESI) for keto form: $m / z[\mathrm{M}+\mathrm{Na}]^{+}$calcd. for $\mathrm{C}_{12} \mathrm{H}_{9} \mathrm{NNaO}_{4}{ }^{+}$: 254.0429; found: 254.0436.

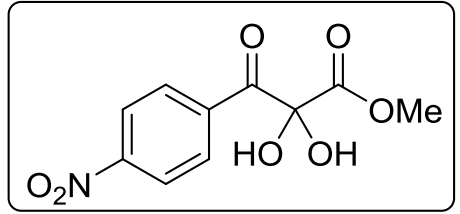

Methyl 2,2-dihydroxy-3-(4-nitrophenyl)-3-oxopropanoate (5'k): white solid (37.3 $\mathrm{mg}, 73 \%)$; $\mathrm{mp} 110.0-111.0{ }^{\circ} \mathrm{C}$ (hexane/ethyl acetate); $\mathrm{R}_{f} 0.40$ (hexane/ethyl acetate $1: 1$ ); ${ }^{1} \mathbf{H}$ NMR (400 MHz, DMSO-d6) $\delta 8.34(\mathrm{~d}, J=8.9 \mathrm{~Hz}, 2 \mathrm{H}, \mathrm{Ar}), 8.25$ (d, $J=8.9 \mathrm{~Hz}, 2 \mathrm{H}, \mathrm{Ar}), 7.87$ (br.s, 2H, OH), 3.66 (s, 3H, Me); ${ }^{13} \mathrm{C}$ NMR (100 MHz, DMSO$\left.d_{6}\right) \delta 193.1,169.8,149.9,137.8,131.0,123.6,94.7,52.5$; HRMS (ESI) for hydrate form: $\mathrm{m} / z$ $[\mathrm{M}+\mathrm{Na}]^{+}$calcd. for $\mathrm{C}_{10} \mathrm{H}_{9} \mathrm{NNaO}_{7}^{+}: 278.0277$; found: 278.0284 .

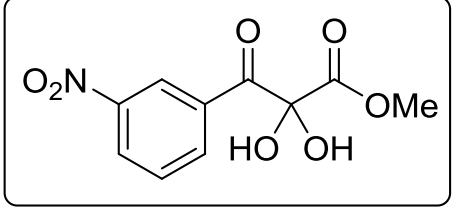

Methyl 2,2-dihydroxy-3-(3-nitrophenyl)-3-oxopropanoate (5'l): white solid (35.2 mg, 69\%); mp 82.0-83.0 ${ }^{\circ} \mathrm{C}$ (hexane/ethyl acetate); $\mathrm{R}_{f} 0.35$ (hexane/ethyl acetate $\left.1: 1\right) ;{ }^{1} \mathbf{H}$ NMR (400 MHz, DMSO- $\left.d_{6}\right) \delta 8.86(\mathrm{t}, J=2.0 \mathrm{~Hz}, 1 \mathrm{H}, \mathrm{Ar}), 8.50-8.47(\mathrm{~m}, 1 \mathrm{H}, \mathrm{Ar})$, 8.43-8.40 (m, 1H, Ar), 7.92 (br.s, 2H, OH), 7.84 (d, J = 8.0 Hz, 1H, Ar), 3.67 (s, 3H, Me); ${ }^{13}$ C NMR (100 MHz, DMSO- $\left.d_{6}\right) \delta$ 192.3, 169.8, 147.7, 135.6, 134.0, 130.5, 127.8, 124.2, 94.8, 52.5; HRMS (ESI) for keto form: $m / z$ [M + Na] $]^{+}$calcd. for $\mathrm{C}_{10} \mathrm{H}_{7} \mathrm{NNaO}_{6}{ }^{+}: 260.0171$; found: 260.0177 .



Methyl 3-(naphthalen-1-yl)-2,3-dioxopropanoate (5m) and methyl 2,2-dihydroxy-3(naphthalen-1-yl)-3-oxopropanoate $\left(5^{\prime} \mathrm{m}\right)$ : 
yellow oil (34.9 mg, 70\%); $\mathrm{R}_{f} 0.30$ (hexane/ethyl acetate 2:1); keto form: hydrate form = 3:2; ${ }^{1} \mathbf{H}$ NMR for keto form (400 MHz, DMSO- $\left.d_{6}\right) \delta 8.33(\mathrm{~d}, J=8.9 \mathrm{~Hz}, 1 \mathrm{H}, \mathrm{Ar}), 8.20(\mathrm{~d}, J=6.1$ $\mathrm{Hz}, 1 \mathrm{H}, \mathrm{Ar}), 8.00$ (d, J=7.9 Hz, 1H, Ar), 7.74-7.56 (m, 4H, Ar), 3.61 (s, 3H, OMe); ${ }^{1} \mathbf{H}$ NMR for hydrate form (400 MHz, DMSO- $\left.d_{6}\right) \delta 8.98(\mathrm{~d}, J=8.5 \mathrm{~Hz}, 1 \mathrm{H}, \mathrm{Ar}), 8.41(\mathrm{~d}, J=8.2 \mathrm{~Hz}$, $1 \mathrm{H}, \mathrm{Ar}), 8.28(\mathrm{~d}, J=6.1 \mathrm{~Hz}, 1 \mathrm{H}, \mathrm{Ar}), 8.13(\mathrm{t}, J=8.1 \mathrm{~Hz}, 2 \mathrm{H}, \mathrm{Ar}), 7.81(\mathrm{t}, J=7.0 \mathrm{~Hz}, 1 \mathrm{H}, \mathrm{Ar})$, 7.74-7.56 (m, 3H, Ar + 2OH), $3.91(\mathrm{~s}, 3 \mathrm{H}, \mathrm{OMe}) ;{ }^{\mathbf{1 3}} \mathbf{C} \mathbf{N M R}$ for keto and hydrate form (100 MHz, DMSO-d $) \delta 198.1,193.6,183.2$, 170.3, 159.8, 137.0, 136.3, 133.5, 133.3, 132.4, 131.6, $130.3,129.9,129.8,129.2,128.7,128.5,127.7,127.4,126.3,125.9,125.2,125.0,124.6$, 124.4, 95.2, 53.6, 52.3; HRMS (ESI) for hydrate form: $m / z[\mathrm{M}+\mathrm{Na}]^{+}$calcd. for $\mathrm{C}_{14} \mathrm{H}_{12} \mathrm{NaO}_{5}{ }^{+}$: 283.0582; found: 283.0589 .

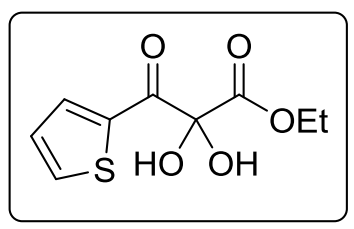

Ethyl 2,2-dihydroxy-3-oxo-3-(thiophen-2-yl)propanoate $\quad\left(5^{\prime} n\right)^{7}$ : yellowish oil (27.2 $\mathrm{mg}, 59 \%$ ); $\mathrm{R}_{f} 0.30$ (hexane/ethyl acetate $2: 1$ ); ${ }^{1} \mathbf{H}$ NMR (400 MHz, DMSO- $\left.d_{6}\right) \delta 8.03(\mathrm{dd}, J=4.9,1.2 \mathrm{~Hz}, 1 \mathrm{H}, \mathrm{Ar}), 7.94$ (dd, $J=3.9,1.2 \mathrm{~Hz}, 1 \mathrm{H}, \mathrm{Ar}), 7.55$ (br.s, 2H, OH), 7.24-7.22 (m, 1H, Ar), $4.10\left(\mathrm{q}, J=7.0 \mathrm{~Hz}, 2 \mathrm{H}, \mathrm{CH}_{2}\right), 1.07$ (t $\left.J=7.1 \mathrm{~Hz}, 3 \mathrm{H}, \mathrm{Me}\right) ;{ }^{13} \mathrm{C}$ NMR (100 MHz, DMSO$\left.d_{6}\right) \delta 187.4,169.3,138.6,135.7,135.3,128.5,94.4,61.2,13.8$; HRMS (ESI) for keto form: $m / z[\mathrm{M}+\mathrm{Na}]^{+}$calcd. for $\mathrm{C}_{9} \mathrm{H}_{8} \mathrm{NaO}_{4} \mathrm{~S}^{+}: 235.0041$; found: 235.0035 .

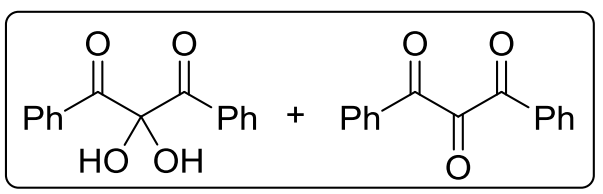

2,2-Dihydroxy-1,3-diphenylpropane-1,3-dione

(5'o) and 1,3-diphenylpropane-1,2,3-trione $(50)^{7}$ : yellowish solid (37.8 mg, 76\%); mp 110.5-111.5 ${ }^{\circ} \mathrm{C}$ (hexane/ethyl acetate); $\mathrm{R}_{f} 0.50$ (hexane/ethyl acetate 2:1); hydrate form:keto form $=3: 2 ;{ }^{1} \mathbf{H}$ NMR for hydrate form (400 MHz, DMSO-d $) \delta 8.02(\mathrm{~d}, J=7.0 \mathrm{~Hz}, 4 \mathrm{H}, \mathrm{Ph}), 7.73$ (br.s, 2H, OH), 7.59 (t, $J=$ $7.4 \mathrm{~Hz}, 2 \mathrm{H}, \mathrm{Ph}), 7.47\left(\mathrm{t}, J=7.7 \mathrm{~Hz}, 4 \mathrm{H}, \mathrm{Ph}\right.$ ); ${ }^{1} \mathbf{H}$ NMR for keto form (400 MHz, DMSO- $d_{6}$ ) $\delta 8.10(\mathrm{~d}, J=7.0 \mathrm{~Hz}, 4 \mathrm{H}, \mathrm{Ph}), 7.84(\mathrm{t}, J=7.5 \mathrm{~Hz}, 2 \mathrm{H}, \mathrm{Ph}), 7.67(\mathrm{t}, J=7.8 \mathrm{~Hz}, 4 \mathrm{H}, \mathrm{Ph}) ;{ }^{13} \mathbf{C}$ NMR for hydrate and keto forms (100 MHz, DMSO- $\left.d_{6}\right) \delta 195.8,191.6,187.9,135.7,133.4$, 133.3, 131.7, 129.94, 129.90, 129.4, 128.4, 96.8; HRMS (ESI) for hydrate form: $\mathrm{m} / z$ [M + $\mathrm{Na}]^{+}$calcd. for $\mathrm{C}_{15} \mathrm{H}_{12} \mathrm{NaO}_{4}^{+}$: 279.0633; found: 279.0620 .

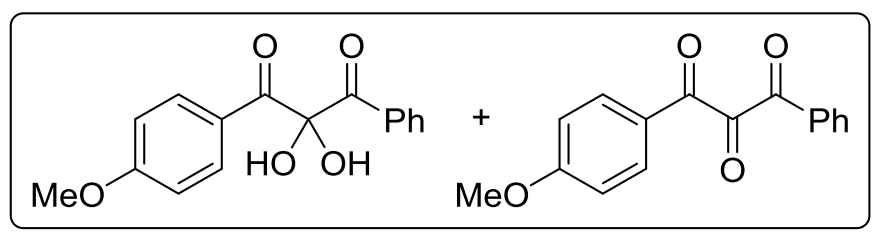

1-(4-Methoxyphenyl)-3-phenylpropane1,2,3-trione (5p) and 2,2-dihydroxy-1-(4methoxyphenyl)-3-phenylpropane-1,3dione (5'p): yellowish oil (44.2 $\mathrm{mg}, 80 \%)$; $\mathrm{R}_{f} 0.35$ (hexane/ethyl acetate 2:1); keto form: hydrate form $=11: 9 ;{ }^{1} \mathbf{H}$ NMR for keto form (400 $\left.\mathrm{MHz}, \mathrm{CDCl}_{3}\right) \delta 8.09(\mathrm{~d}, J=8.9 \mathrm{~Hz}, 2 \mathrm{H}, \mathrm{Ar}), 7.96-7.93(\mathrm{~m}, 2 \mathrm{H}, \mathrm{Ar}), 7.70(\mathrm{t}, J=7.4 \mathrm{~Hz}, 1 \mathrm{H}$, Ar), $7.55(\mathrm{t}, J=7.8 \mathrm{~Hz}, 2 \mathrm{H}, \mathrm{Ar}), 7.02$ (d, $J=8.9 \mathrm{~Hz}, 2 \mathrm{H}, \mathrm{Ar}), 3.91$ (s, 3H, OMe); ${ }^{1} \mathbf{H}$ NMR 
for hydrate form (400 MHz, $\left.\mathrm{CDCl}_{3}\right) \delta 8.06(\mathrm{~d}, J=7.1 \mathrm{~Hz}, 2 \mathrm{H}, \mathrm{Ar}), 7.54-7.43$ (m, 3H, Ar), 7.36 (t, $J=7.9 \mathrm{~Hz}, 2 \mathrm{H}, \mathrm{Ar}), 6.82$ (d, $J=9.0 \mathrm{~Hz}, 2 \mathrm{H}, \mathrm{Ar}), 5.95$ (br.s, 2H, OH), 3.80 (s, 3H, $\mathrm{OMe}) ;{ }^{13} \mathbf{C} \mathbf{N M R}$ for keto and hydrate forms (100 $\left.\mathrm{MHz}, \mathrm{CDCl}_{3}\right) \delta$ 194.6, 193.2, 192.3, 190.5, 189.0, 165.6, 164.9, 135.4, 134.7, 134.2, 133.1, 133.0, 130.4, 130.3, 129.4, 129.2, 128.9, 125.3, 125.0, 114.6, 114.3, 94.1, 55.8, 55.7; HRMS (ESI) for keto form: $\mathrm{m} / z[\mathrm{M}+\mathrm{Na}]^{+}$calcd. for $\mathrm{C}_{16} \mathrm{H}_{12} \mathrm{NaO}_{4}^{+}$: 291.0633; found: 291.0639 .

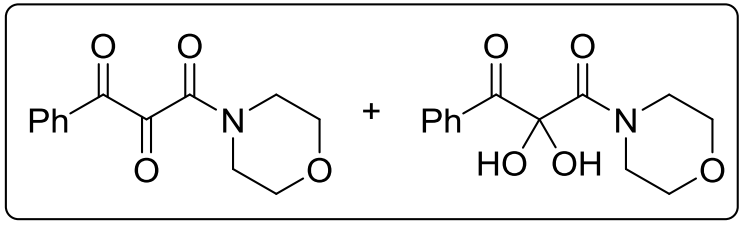

\section{1-Morpholino-3-phenylpropane-1,2,3-trione} (5q) and 2,2-dihydroxy-1-morpholino-3phenylpropane-1,3-dione $\left(\mathbf{5}^{\prime} \mathbf{q}\right)^{7}$ : yellowish solid

(46.2 mg, 92\%); mp 88.0-89.5 ${ }^{\circ} \mathrm{C}$ (hexane/ethyl acetate); $\mathrm{R}_{f} 0.30$ (hexane/ethyl acetate $2: 1$ ); keto form:hydrate form $=10: 3 ;{ }^{1} \mathbf{H} \mathbf{N M R}$ for keto form (400 MHz, $\left.\mathrm{CDCl}_{3}\right) \delta 8.05(\mathrm{~d}, J=6.9 \mathrm{~Hz}, 2 \mathrm{H}, \mathrm{Ph}), 7.68(\mathrm{t}, J=7.5 \mathrm{~Hz}, 1 \mathrm{H}, \mathrm{Ph}), 7.53(\mathrm{t}, J$ $=7.8 \mathrm{~Hz}, 2 \mathrm{H}, \mathrm{Ph}), 3.80-3.77\left(\mathrm{~m}, 4 \mathrm{H}, \mathrm{CH}_{2}\right), 3.66-3.59\left(\mathrm{~m}, 3 \mathrm{H}, \mathrm{CH}_{2}\right), 3.35-3.22\left(\mathrm{~m}, 1 \mathrm{H}, \mathrm{CH}_{2}\right)$; ${ }^{13} \mathbf{C}$ NMR for keto form $\left(100 \mathrm{MHz}, \mathrm{CDCl}_{3}\right) \delta 192.0,185.5,164.3,135.5,131.9,130.5,129.1$, 67.0, 66.6, 46.3, 42.2; ${ }^{1} \mathbf{H}$ NMR for hydrate form (400 MHz, $\left.\mathrm{CDCl}_{3}\right) \delta 8.04-8.01(\mathrm{~m}, 2 \mathrm{H}, \mathrm{Ph}$ ), 7.62 (t, $J=7.5 \mathrm{~Hz}, 1 \mathrm{H}, \mathrm{Ph}), 7.46$ (t, $J=7.9 \mathrm{~Hz}, 2 \mathrm{H}, \mathrm{Ph}), 5.89$ (br.s, 2H, OH), 3.72-3.70 (m, $\left.6 \mathrm{H}, \mathrm{CH}_{2}\right), 3.55-3.53\left(\mathrm{~m}, 2 \mathrm{H}, \mathrm{CH}_{2}\right) ;{ }^{13} \mathbf{C ~ N M R}$ for hydrate form $\left(100 \mathrm{MHz}, \mathrm{CDCl}_{3}\right) \delta 194.3$, 167.5, 134.9, 130.6, 130.1, 129.0, 91.5, 66.5, 66.2, 46.4, 44.2; HRMS (ESI) for keto form: $m / z[\mathrm{M}+\mathrm{Na}]^{+}$calcd. for $\mathrm{C}_{13} \mathrm{H}_{13} \mathrm{NNaO}_{4}{ }^{+}: 270.0742$; found: 270.0737 .

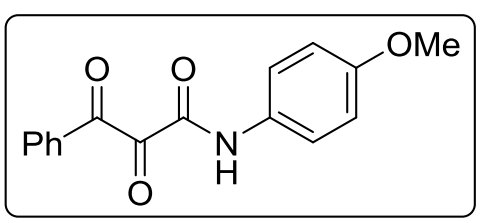

$N$-(4-Methoxyphenyl)-2,3-dioxo-3-phenylpropanamide (5r) ${ }^{9}$ : yellowish oil (40.2 mg, 72\%); $\mathrm{R}_{f} 0.35$ (hexane/ethyl acetate $2: 1$ ); ${ }^{1} \mathbf{H}$ NMR (400 MHz, $\left.\mathrm{CDCl}_{3}\right) \delta 8.60$ (br. s, 1H, NH), 7.91 (d, $J=$ $7.0 \mathrm{~Hz}, 2 \mathrm{H}, \mathrm{Ph}), 7.69(\mathrm{t}, J=7.5 \mathrm{~Hz}, 1 \mathrm{H}, \mathrm{Ph}), 7.60(\mathrm{~d}, J=9.0 \mathrm{~Hz}$, 2H, Ar), 7.53 (t, $J=7.8 \mathrm{~Hz}, 2 \mathrm{H}, \mathrm{Ph}), 6.91$ (d, $J=9.0 \mathrm{~Hz}, 2 \mathrm{H}, \mathrm{Ar}), 3.81$ (s, 3H, Me); ${ }^{13} \mathbf{C}$ NMR $\left(100 \mathrm{MHz}, \mathrm{CDCl}_{3}\right) \delta 193.8,189.5,157.6,156.3,135.5,132.3,129.6,129.3,129.1,121.6$, 114.6, 55.6; HRMS (ESI) for keto form: $m / z[\mathrm{M}+\mathrm{Na}]^{+}$calcd. for $\mathrm{C}_{16} \mathrm{H}_{13} \mathrm{NNaO}_{4}{ }^{+}$: 306.0742 ; found: 306.0735 .

\subsection{One-pot synthesis of azaheterocyclic compounds 6-9}

$\mathrm{Ph}_{3} \mathrm{PAuNTf}_{2}(7.4 \mathrm{mg}, 10.0 \mu \mathrm{mol}, 5 \mathrm{~mol} \%$ ) was added to the solution of 2,6-dichloropyridine $N$-oxide 3d (82.0 mg, $0.5 \mathrm{mmol}, 2.5$ equiv) and ethyl non-2-ynoate (36.5 mg, $0.2 \mathrm{mmol})$ or 4-phenylbut-3-yn2-one $(28.8 \mathrm{mg}, 0.2 \mathrm{mmol})$ in $\mathrm{PhCF}_{3}(1.0 \mathrm{~mL})$. The resulting mixture was stirred at room temperature for $1 \mathrm{~h}$. Then $o$-phenylenediamine $(32.4 \mathrm{mg}, 0.3 \mathrm{mmol}, 1.5$ equiv) was added and the 
resulted mixture was stirred at $60{ }^{\circ} \mathrm{C}$ for $5 \mathrm{~h}$. Upon cooling to r.t.,. the solvent was removed in vасио and the residue was purified by silica gel chromatography eluting with hexane/EtOAc (gradient from 8:1 to 4:1) to afford quinoxalines 6.

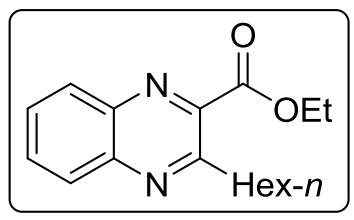

Ethyl 3-hexylquinoxaline-2-carboxylate (6a): yellowish oil $(36.1 \mathrm{mg}$, $63 \%) ; \mathrm{R}_{f} 0.50$ (hexane/ethyl acetate 4:1); ${ }^{1} \mathbf{H}$ NMR (400 MHz, $\mathrm{CDCl}_{3}$ ) $\delta 8.15(\mathrm{~d}, J=6.9 \mathrm{~Hz}, 1 \mathrm{H}, \mathrm{Ar}), 8.07(\mathrm{~d}, J=6.9 \mathrm{~Hz}, 1 \mathrm{H}, \mathrm{Ar}), 7.81(\mathrm{t}, J=$ $6.8 \mathrm{~Hz}, 1 \mathrm{H}, \mathrm{Ar}), 7.74(\mathrm{t}, J=6.8 \mathrm{~Hz}, 1 \mathrm{H}, \mathrm{Ar}), 4.55(\mathrm{q}, J=7.1 \mathrm{~Hz}, 2 \mathrm{H}$, $\left.\mathrm{OCH}_{2}\right), 3.23-3.20\left(\mathrm{~m}, 2 \mathrm{H}, \mathrm{CH}_{2}\right), 1.86-1.78\left(\mathrm{~m}, 2 \mathrm{H}, \mathrm{CH}_{2}\right), 1.50-1.43(\mathrm{~m}, 4 \mathrm{H}), 1.38-1.25(\mathrm{~m}$, 5H), 0.90-0.87 (m, 3H, Me); $\left.{ }^{13} \mathbf{C ~ N M R ~ ( 1 0 0 ~ M H z , ~} \mathrm{CDCl}_{3}\right) \delta 166.1,156.3,145.2,142.7$, 139.9, 131.6, 129.8 (2C), 128.8, 62.6, 36.2, 31.8, 29.6, 29.5, 27.7, 14.4, 14.2; HRMS (ESI): $m / z[\mathrm{M}+\mathrm{Na}]^{+}$calcd. for $\mathrm{C}_{17} \mathrm{H}_{22} \mathrm{~N}_{2} \mathrm{NaO}_{2}{ }^{+}: 309.1579$; found: 309.1568 .

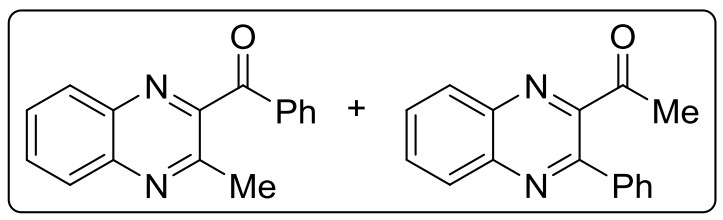

(3-Methylquinoxalin-2-yl)(phenyl)methanone $(6 b)^{10}$ and 1-(3-phenylquinoxalin-2-yl)ethan-1one $\left(6^{\prime}\right)$ : yellowish oil (37.2 $\left.\mathrm{mg}, 75 \%\right) ; \mathrm{R}_{f} 0.35$ (hexane/ethyl acetate 2:1); mixture $=4: 1 ;{ }^{1} \mathbf{H}$ NMR for $6 \mathbf{b}\left(400 \mathrm{MHz}, \mathrm{CDCl}_{3}\right) \delta 8.09(\mathrm{~d}, J=7.9 \mathrm{~Hz}, 2 \mathrm{H}, \mathrm{Ar}), 7.97-7.95$ (m, 2H, Ar), 7.88-7.81 (m, 1H, Ar), 7.78-7.73 (m, 1H, Ar), 7.67-7.63 (m, 1H, Ar), 7.52-7.48 (m, 2H, Ar), 2.81 (s, $3 \mathrm{H}, \mathrm{Me}) ;{ }^{13} \mathbf{C} \mathbf{N M R}$ for $\mathbf{6 b}\left(100 \mathrm{MHz}, \mathrm{CDCl}_{3}\right) \delta 194.1,152.4,151.0,142.2,139.5,135.7$, 134.3, 131.4, 130.8, 129.9, 129.6, 128.8, 128.7, 22.9; ${ }^{\mathbf{1}} \mathbf{H} \mathbf{N M R}$ for $\mathbf{6} \mathbf{b}^{\prime}\left(400 \mathrm{MHz}, \mathrm{CDCl}_{3}\right) \delta$ $8.17(\mathrm{~d}, J=8.1 \mathrm{~Hz}, 2 \mathrm{H}, \mathrm{Ar}), 7.88-7.81$ (m, 2H, Ar), 7.67-7.63 (m, 2H, Ar), 7.52-7.48 (m, $3 \mathrm{H}, \mathrm{Ar}), 2.76$ (s, 3H, Me); ${ }^{13} \mathbf{C} \mathbf{N M R}$ for 6b' (100 $\left.\mathbf{M H z}, \mathrm{CDCl}_{3}\right) \delta 201.2,152.6,150.2,142.5$, 139.9, 138.2, 131.9, 130.5, 129.7, 129.52, 129.48, 129.1, 128.7, 28.6;HRMS (ESI): $m / z[M+$ $\mathrm{Na}^{+}$calcd. for $\mathrm{C}_{16} \mathrm{H}_{12} \mathrm{~N}_{2} \mathrm{NaO}^{+}$: 271.0847; found: 271.0855 .

$\mathrm{Ph}_{3} \mathrm{PAuNTf}_{2}(7.4 \mathrm{mg}, 10.0 \mu \mathrm{mol}, 5 \mathrm{~mol} \%)$ was added to the solution of ethyl 3-phenylpropiolate (34.8 mg, $0.2 \mathrm{mmol}$ ) and 2,6-dichloropyridine $N$-oxide $3 \mathbf{d}$ (82.0 mg, $0.5 \mathrm{mmol}, 2.5$ equiv) in $\mathrm{PhCF}_{3}$ $(1.0 \mathrm{~mL})$. The resulting mixture was stirred at room temperature for $1 \mathrm{~h}$. Then $p$-anisidine (29.6 $\mathrm{mg}, 0.24 \mathrm{mmol}, 1.2$ equiv), cyclohexanone (39.3 mg, $0.4 \mathrm{mmol}, 2.0$ equiv) and benzoic acid (4.9 mg, $40.0 \mu \mathrm{mol}, 20 \mathrm{~mol} \%$ ) were added and the resulted mixture was stirred at $65^{\circ} \mathrm{C}$ for $36 \mathrm{~h}$. Upon cooling to r.t.,. the solvent was removed in vacuo and the residue was purified by silica gel chromatography eluting with hexane/EtOAc (gradient from 8:1 to 4:1) to afford indole 8. 


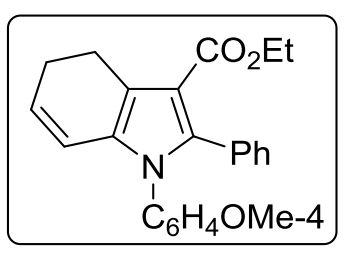

Ethyl

1-(4-methoxyphenyl)-2-phenyl-4,5-dihydro-1 $H$-indole-3-

carboxylate $(7)^{11}$ : yellow solid $(38.9 \mathrm{mg}, 52 \%)$; mp $153.0-155.0{ }^{\circ} \mathrm{C}$ (hexane/ethyl acetate); $\mathrm{R}_{f} 0.50$ (hexane/ethyl acetate 4:1); ${ }^{1} \mathbf{H}$ NMR (400

$\left.\mathrm{MHz} \mathrm{CDCl}_{3}\right) \delta 7.19-7.14(\mathrm{~m}, 5 \mathrm{H}, \mathrm{Ar}), 6.95(\mathrm{~d}, J=8.9 \mathrm{~Hz}, 2 \mathrm{H}, \mathrm{Ar}), 6.76$ $(\mathrm{d}, J=8.9 \mathrm{~Hz}, 2 \mathrm{H}, \mathrm{Ar}), 6.01(\mathrm{~d}, J=9.9 \mathrm{~Hz}, 1 \mathrm{H}, \mathrm{CH}), 5.74-5.69(\mathrm{~m}, 1 \mathrm{H}, \mathrm{CH}), 4.13$ (q, $J=7.1$ $\left.\mathrm{Hz}, 2 \mathrm{H}, \mathrm{OCH}_{2}\right), 3.76(\mathrm{~s}, 3 \mathrm{H}, \mathrm{OMe}), 3.04\left(\mathrm{t}, J=9.2 \mathrm{~Hz}, 2 \mathrm{H}, \mathrm{CH}_{2}\right), 2.47-2.41\left(\mathrm{~m}, 2 \mathrm{H}, \mathrm{CH}_{2}\right)$, $1.13(\mathrm{t}, J=7.1 \mathrm{~Hz}, 3 \mathrm{H}, \mathrm{Me}) ;{ }^{13} \mathbf{C} \mathbf{N M R}\left(100 \mathrm{MHz}, \mathrm{CDCl}_{3}\right) \delta 165.5,158.8,137.8,132.1,131.3$, $130.8,129.9,129.3,127.5,127.4,124.6,119.2,117.3,114.0,112.0,59.4,55.5,24.2,21.3$, 14.3; HRMS (ESI): $m / z[\mathrm{M}+\mathrm{H}]^{+}$calcd. for $\mathrm{C}_{24} \mathrm{H}_{24} \mathrm{NO}_{3}{ }^{+}: 374.1756$; found: 374.1756 .

$\mathrm{Ph}_{3} \mathrm{PAuNTf}_{2}(7.4 \mathrm{mg}, 10.0 \mu \mathrm{mol}, 5 \mathrm{~mol} \%)$ was added to the solution of ethyl 3-phenylpropiolate (34.8 mg, $0.2 \mathrm{mmol})$ and 2,6-dichloropyridine $N$-oxide $3 \mathbf{d}$ (82.0 mg, $0.5 \mathrm{mmol}, 2.5$ equiv) in $\mathrm{PhCF}_{3}$ $(1.0 \mathrm{~mL})$. The resulting mixture was stirred at room temperature for $1 \mathrm{~h}$. Then $p$-anisidine $(29.6$ mg, $0.24 \mathrm{mmol}, 1.2$ equiv), 5-phenylcyclohexane-1,3-dione (75.3 mg, $0.4 \mathrm{mmol}, 2.0$ equiv) and trifluoroacetic acid (2.3 mg, $20.0 \mu \mathrm{mol}, 10 \mathrm{~mol} \%)$ were added and the resulted mixture was stirred at $65{ }^{\circ} \mathrm{C}$ for $16 \mathrm{~h}$. Upon cooling to r.t.,. the solvent was removed in vacuo and the residue was purified by silica gel chromatography eluting with hexane/EtOAc (4:1) to afford indole $\mathbf{8}$.

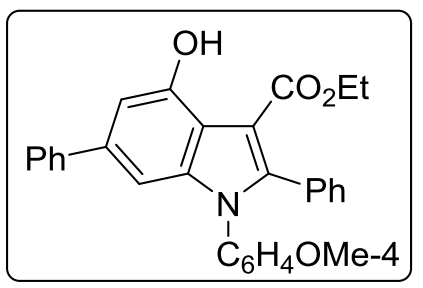

Ethyl 4-hydroxy-1-(4-methoxyphenyl)-2,6-diphenyl-1H-indole3-carboxylate (8): beige solid $(42.8 \mathrm{mg}, 60 \%)$; $\mathrm{mp} 229.0-230.0{ }^{\circ} \mathrm{C}$ (dec., hexane/ethyl acetate); $\mathrm{R}_{f} 0.40$ (hexane/ethyl acetate $4: 1$ ); ${ }^{1} \mathbf{H}$ NMR $\left(400 \mathrm{MHz}, \mathrm{CDCl}_{3}\right) \delta 11.47(\mathrm{~s}, 1 \mathrm{H}, \mathrm{OH}), 7.58(\mathrm{~d}, J=7.0 \mathrm{~Hz}$, 2H, Ar), 7.38 (t, $J=7.5 \mathrm{~Hz}, 2 \mathrm{H}, \mathrm{Ar}), 7.31-7.21$ (m, 6H, Ar), 7.11$7.08(\mathrm{~m}, 3 \mathrm{H}, \mathrm{Ar}), 6.84(\mathrm{~d}, J=8.9 \mathrm{~Hz}, 2 \mathrm{H}, \mathrm{Ar}), 6.79$ (d, $J=1.4 \mathrm{~Hz}, 1 \mathrm{H}, \mathrm{Ar}), 4.12$ (q, $J=7.1$ $\left.\mathrm{Hz}, 2 \mathrm{H}, \mathrm{OCH}_{2}\right), 3.79(\mathrm{~s}, 3 \mathrm{H}, \mathrm{OMe}), 0.90(\mathrm{t}, J=7.1 \mathrm{~Hz}, 3 \mathrm{H}, \mathrm{Me}) ;{ }^{13} \mathbf{C ~ N M R}\left(100 \mathrm{MHz}, \mathrm{CDCl}_{3}\right)$ $\delta 168.6,159.3,151.7,146.8,141.7,140.7,139.2,131.9,130.8,129.7,129.3,128.7,128.5$, 127.5, 127.4, 127.1 1 14.6, 114.5, 107.8, 105.6, 100.9, 61.1, 55.5, 13.5; HRMS (ESI): $m / z$ [M $+\mathrm{H}]^{+}$calcd. for $\mathrm{C}_{30} \mathrm{H}_{26} \mathrm{NO}_{4}^{+}$: 464.1856; found: 464.1845 .

$\mathrm{Ph}_{3} \mathrm{PAuNTf}_{2}(7.4 \mathrm{mg}, 10.0 \mu \mathrm{mol}, 5 \mathrm{~mol} \%)$ was added to the solution of ethyl 3-phenylpropiolate (34.8 mg, $0.2 \mathrm{mmol}$ ) and 2,6-dichloropyridine $N$-oxide $3 \mathbf{d}$ (82.0 mg, $0.5 \mathrm{mmol}, 2.5$ equiv) in $\mathrm{PhCF}_{3}$ $(1.0 \mathrm{~mL})$. The resulting mixture was stirred at room temperature for $1 \mathrm{~h}$. Then ethylenediamine diacetate (54.1 mg, $0.3 \mathrm{mmol}, 1.5$ equiv) was added and the resulted mixture was stirred at $80{ }^{\circ} \mathrm{C}$ for $5 \mathrm{~h}$. Upon cooling to r.t.,. the solvent was removed in vacuo and the residue was purified by silica gel chromatography eluting with hexane/EtOAc (4:1) to afford pyrazine 7. 


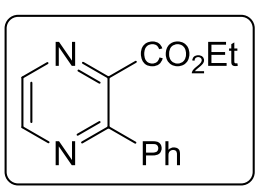

Ethyl 3-phenylpyrazine-2-carboxylate (9): yellow oil (39.3 mg, 86\%); $\mathrm{R}_{f}$ 0.20 (hexane/ethyl acetate 4:1); ${ }^{1} \mathbf{H}$ NMR (400 $\left.\mathrm{MHz} \mathrm{CDCl}_{3}\right) \delta 8.74(\mathrm{~d}, J=$ $2.4 \mathrm{~Hz}, 1 \mathrm{H}, \mathrm{Ar}), 8.59$ (d, $J=2.4 \mathrm{~Hz}, 1 \mathrm{H}, \mathrm{Ar}), 7.65-7.60$ (m, 2H, Ar), 7.497.45 (m, 3H, Ar), 4.29 (q, $\left.J=7.1 \mathrm{~Hz}, 2 \mathrm{H}, \mathrm{CH}_{2}\right), 1.17(\mathrm{t}, J=7.2 \mathrm{~Hz}, 3 \mathrm{H}, \mathrm{Me}) ;{ }^{13} \mathbf{C}$ NMR (100 $\left.\mathrm{MHz}, \mathrm{CDCl}_{3}\right) \delta 166.4,153.7,145.2,145.0 .141 .9,137.3,129.8,128.7,128.6,62.3,13.9$; HRMS (ESI): $m / z[\mathrm{M}+\mathrm{Na}]^{+}$calcd. for $\mathrm{C}_{13} \mathrm{H}_{12} \mathrm{~N}_{2} \mathrm{NaO}_{2}{ }^{+}: 251.0796$; found: 251.0805 .

$\mathrm{Ph}_{3} \mathrm{PAuNTf}_{2}(7.4 \mathrm{mg}, 10.0 \mu \mathrm{mol}, 5 \mathrm{~mol} \%)$ was added to the solution of ethyl 3-phenylpropiolate (34.8 mg, $0.2 \mathrm{mmol}$ ) and 2,6-dichloropyridine $N$-oxide $\mathbf{3 d}$ (82.0 mg, $0.5 \mathrm{mmol}, 2.5$ equiv) in $\mathrm{PhCF}_{3}$ $(1.0 \mathrm{~mL})$. The resulting mixture was stirred at room temperature for $1 \mathrm{~h}$. Then $o$-aminophenol ( $32.7 \mathrm{mg}, 0.3 \mathrm{mmol}, 1.5$ equiv) was added and the resulted mixture was stirred at $60^{\circ} \mathrm{C}$ for $2 \mathrm{~h}$. Upon cooling to r.t.,. the solvent was removed in vacuo and the residue was purified by silica gel chromatography eluting with hexane/EtOAc (4:1) to afford oxazine 7.

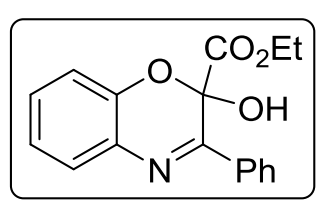

Ethyl 2-hydroxy-3-phenyl-2H-benzo[b][1,4]oxazine-2-carboxylate (10): beige solid (43.4 mg, 73\%); mp 97.0-99.0 ${ }^{\circ} \mathrm{C}$ (hexane/ethyl acetate); $\mathrm{R}_{f} 0.40$ (hexane/ethyl acetate 4:1); ${ }^{\mathbf{1}} \mathbf{H} \mathbf{N M R}\left(400 \mathrm{MHz}, \mathrm{CDCl}_{3}\right) \delta 7.85-$ 7.83 (m, 2H, Ar), 7.61 (dd, $J=7.8,1.6 \mathrm{~Hz}, 1 \mathrm{H}, \mathrm{Ar}), 7.46-7.38$ (m, 3H, Ar), 7.27 (td, $J=7.7$, $1.7 \mathrm{~Hz}, 1 \mathrm{H}, \mathrm{Ar}), 7.13$ (td, $J=7.6,1.4 \mathrm{~Hz}, 1 \mathrm{H}, \mathrm{Ar}), 7.02$ (dd, $J=8.0,1.4 \mathrm{~Hz}, 1 \mathrm{H}, \mathrm{Ar}), 5.09$ (s, $1 \mathrm{H}, \mathrm{OH}), 4.23-4.15\left(\mathrm{~m}, 1 \mathrm{H}, \mathrm{CH}_{2}\right), 4.09-4.01\left(\mathrm{~m}, 1 \mathrm{H}, \mathrm{CH}_{2}\right), 0.86(\mathrm{t}, J=7.1 \mathrm{~Hz}, 3 \mathrm{H}, \mathrm{Me}) ;{ }^{13} \mathrm{C}$ NMR $\left(100 \mathrm{MHz}, \mathrm{CDCl}_{3}\right) \delta 169.1,154.5,142.3,135.9 .131 .4,130.6,129.6,128.6,128.5$, 127.4, 123.1, 116.5, 89.7, 63.7, 13.4; HRMS (ESI): $m / z[M+\mathrm{H}]^{+}$calcd. for $\mathrm{C}_{17} \mathrm{H}_{16} \mathrm{NO}_{4}{ }^{+}$: 298.1079; found: 298.1079.

For compounds $\mathbf{5 b} \mathbf{b}-\mathbf{d}, \mathbf{g}, \mathbf{h}, \mathbf{j}, \mathbf{o}, \mathbf{q}, \mathbf{r}, \mathbf{6 b}$ and $\mathbf{7}$ the ${ }^{1} \mathrm{H},{ }^{13} \mathrm{C}$, HRMS spectra and melting points are consistent with previously reported literature.

\section{3. $\quad$ Control experiment}

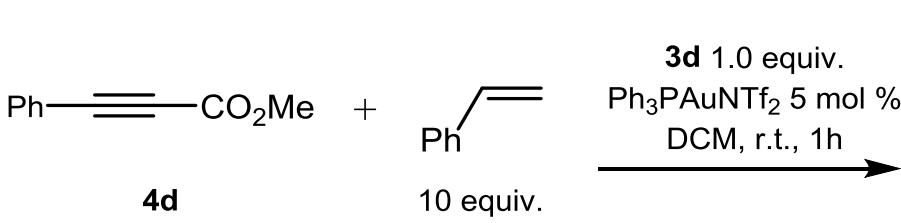

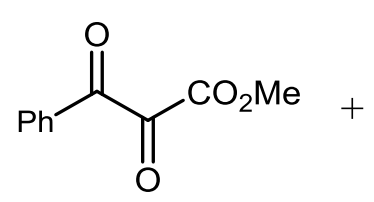

$5 d, 40 \%$



$11,5 \%$

$\mathrm{Ph}_{3} \mathrm{PAuNTf}_{2}(7.4 \mathrm{mg}, 10.0 \mu \mathrm{mol}, 5 \mathrm{~mol} \%$ ) was added to the solution of methyl 3-phenylpropiolate 4d (32.0 mg, $0.2 \mathrm{mmol}$ ), styrene (208.3 mg, $2.0 \mathrm{mmol}, 10.0$ equiv) and 2,6-dichloropyridine $N$-oxide 3d $(32.8 \mathrm{mg}, 0.2 \mathrm{mmol}, 1.0$ equiv) in DCM $(1.0 \mathrm{~mL})$. The resulting mixture was stirred at room 
temperature for $1 \mathrm{~h}$. Spectral characteristics ( ${ }^{1} \mathrm{H}$ NMR, HRMS (ESI)) of methyl 1-benzoyl-2phenylcyclopropane-1-carboxylate (11) are consistent with previously reported literature. ${ }^{12}$

(1) Rassadin, V. A.; Boyarskiy, V. P.; Kukushkin, V. Y. Org. Lett. 2015, 17, 3502-3505.

(2) Rassadin, V. A.; Zimin, D. P.; Raskil'dina, G. Z.; Ivanov, A. Y.; Boyarskiy, V. P.; Zlotskii, S. S.; Kukushkin, V. Y. Green Chem. 2016, 18, 6630-6636.

(3) Rong, D.; Phillips, V. A.; Sánchez, R.; Ángeles, M.; Wheelhouse, R. T. Tetrahedron Lett. 2008, 49, 6931-6933.

(4) Dubovtsev, A. Y.; Dar'in, D. V.; Kukushkin, V. Y. Adv. Synth. Catal. 2019, 361; doi: 10.1002/adsc.201900097.

(5) Liu, J.; Wei, W.; Zhao, T.; Liu, X.; Wu, J.; Yu, W.; Chang, J. J. Org. Chem. 2016, 81, 9326-9336.

(6) Nilov, D. I.; Vasilyev, A. V. Tetrahedron Lett. 2015, 61, 11333-11344.

(7) Cui, J.; Duan, Y. N.; Yu, J.; Zhang, C. Org. Chem. Front. 2016, 3, 1686-1690.

(8) Sha, Q.; Doyle, M. P. Chem. Commun. 2015, 52, 108-111.

(9) Liu, Y.; Zhang, Z.; Wan, Y.; Zhang, G.; Li, Z.; Bi, J.; Liu, T.; Liu, Q. J. Org. Chem. 2017, 82, $3901-3907$.

(10) Sharma, S.; Kumar, M.; Vishwakarma, R. A.; Verma, M. K.; Singh, P. P. J. Org. Chem. 2018, 83, 1242012431.

(11) Sha, Q.; Arman, H.; Doyle, M. P. Org. Lett. 2015, 17, 3876-3879.

(12) Marcoux, D.; Goudreau, R.; Charette, B. J. Org. Chem. 2009, 84, 8939-8955. 
<smiles>CCOC(=O)C(O)(O)C(=O)c1ccc(F)cc1</smiles>

$5 a$
<smiles>CCOC(=O)C(O)(O)C(=O)c1ccc(F)cc1</smiles>

$5 a$

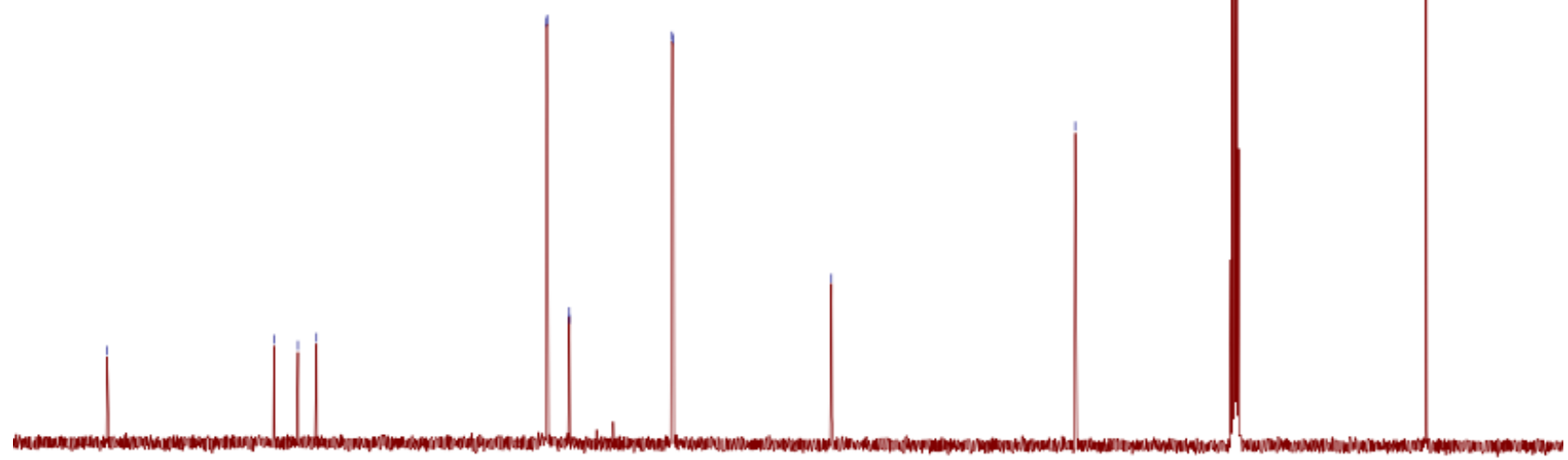

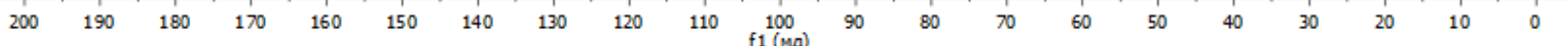




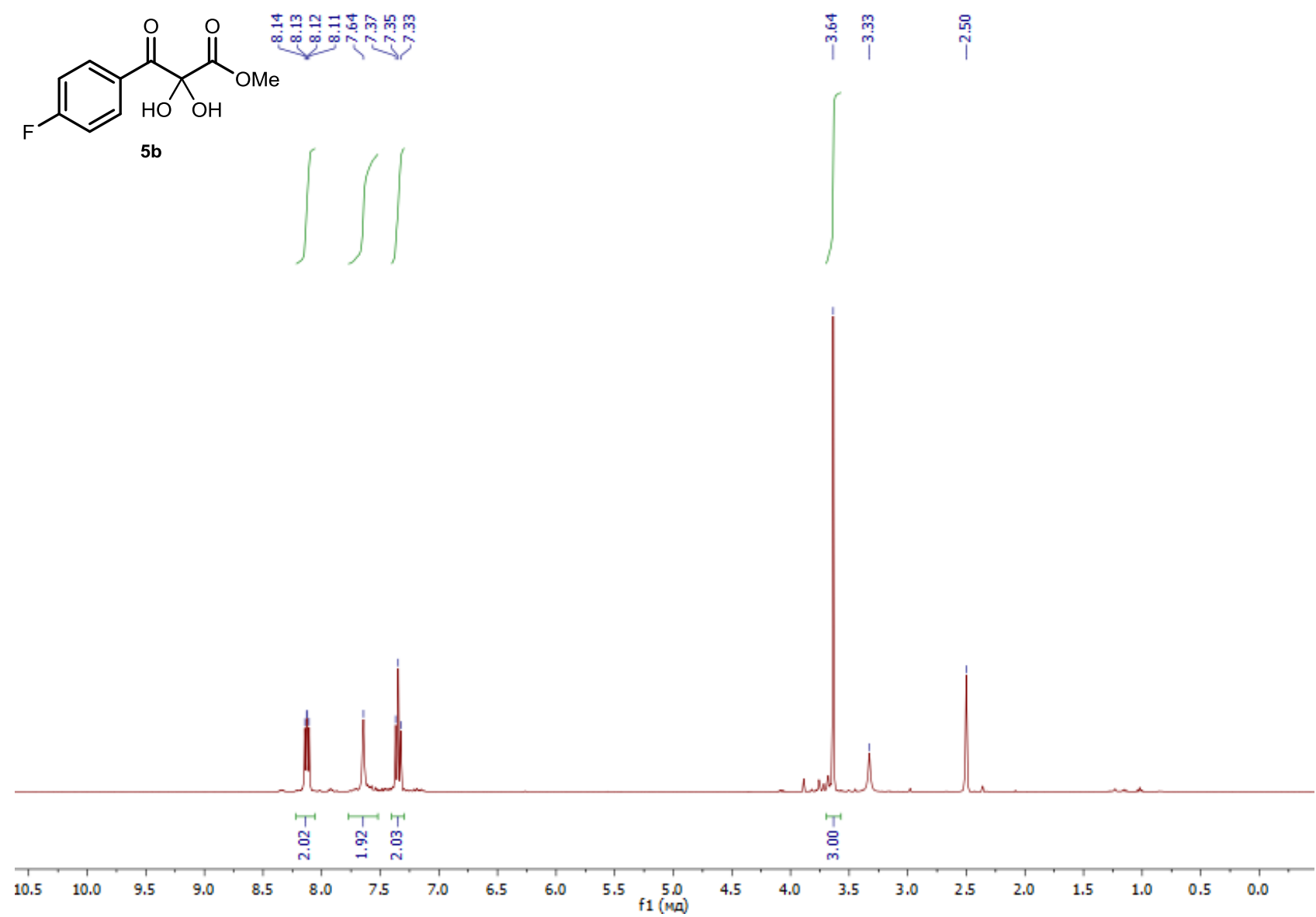<smiles>COC(=O)C(O)(O)C(=O)c1ccc(F)cc1</smiles>

5b








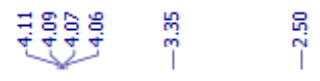

mag
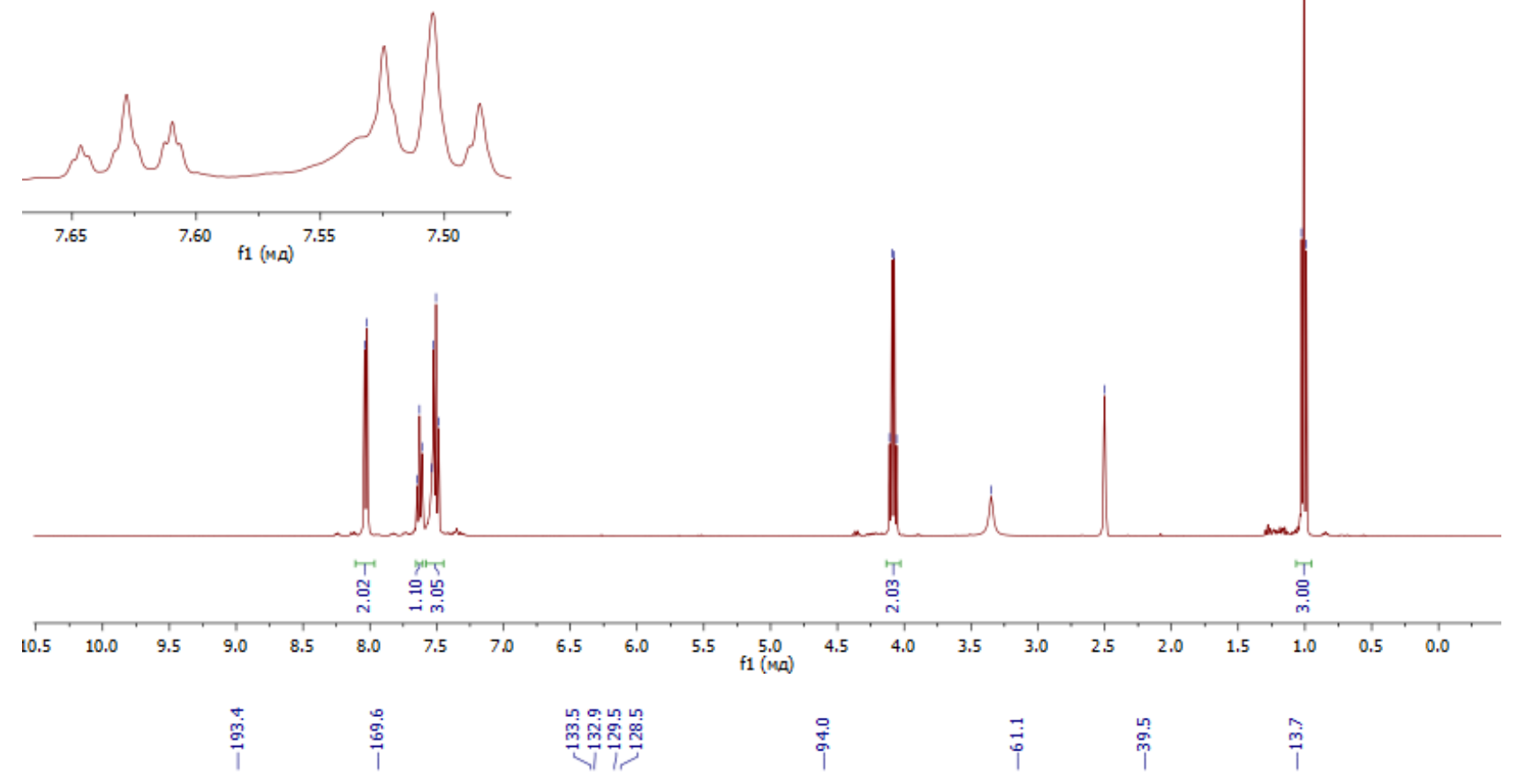<smiles>CCOC(=O)C(O)(O)C(=O)c1ccccc1</smiles>
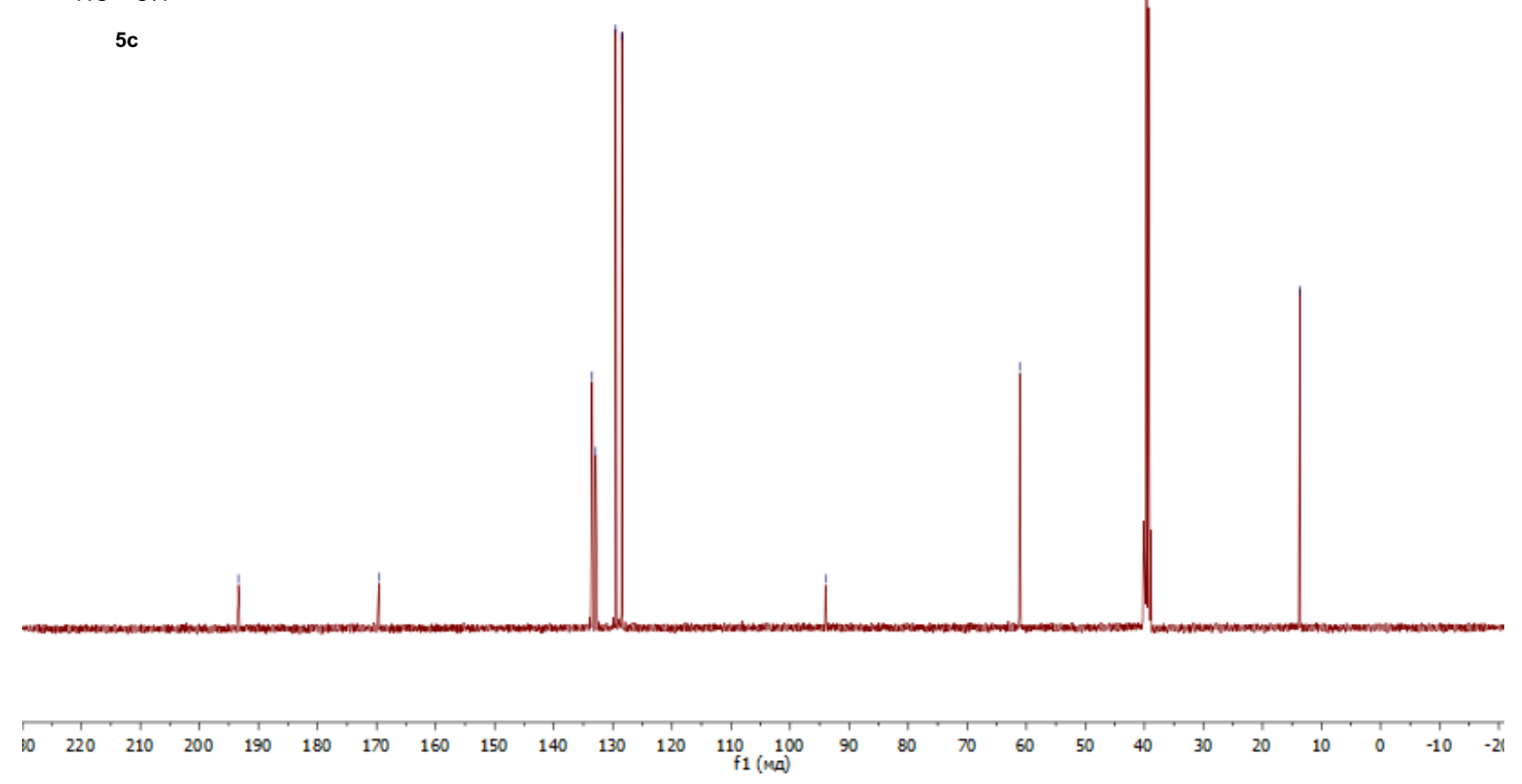

14 
<smiles>COC(=O)C(O)(O)C(=O)c1ccccc1</smiles>

$5 d$
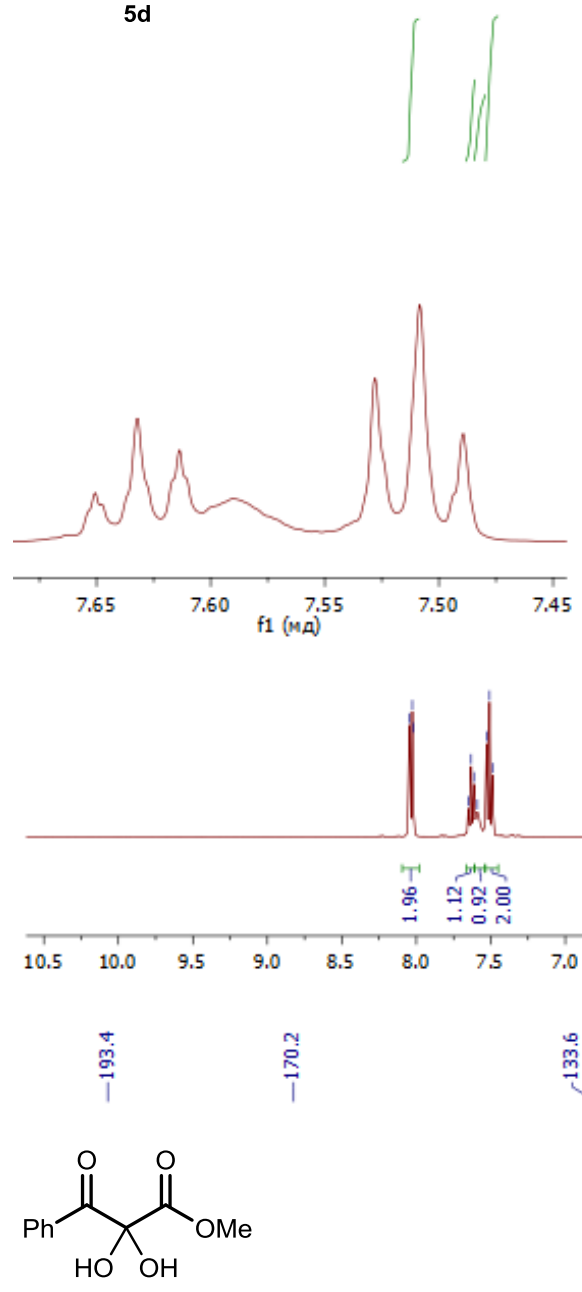

5d
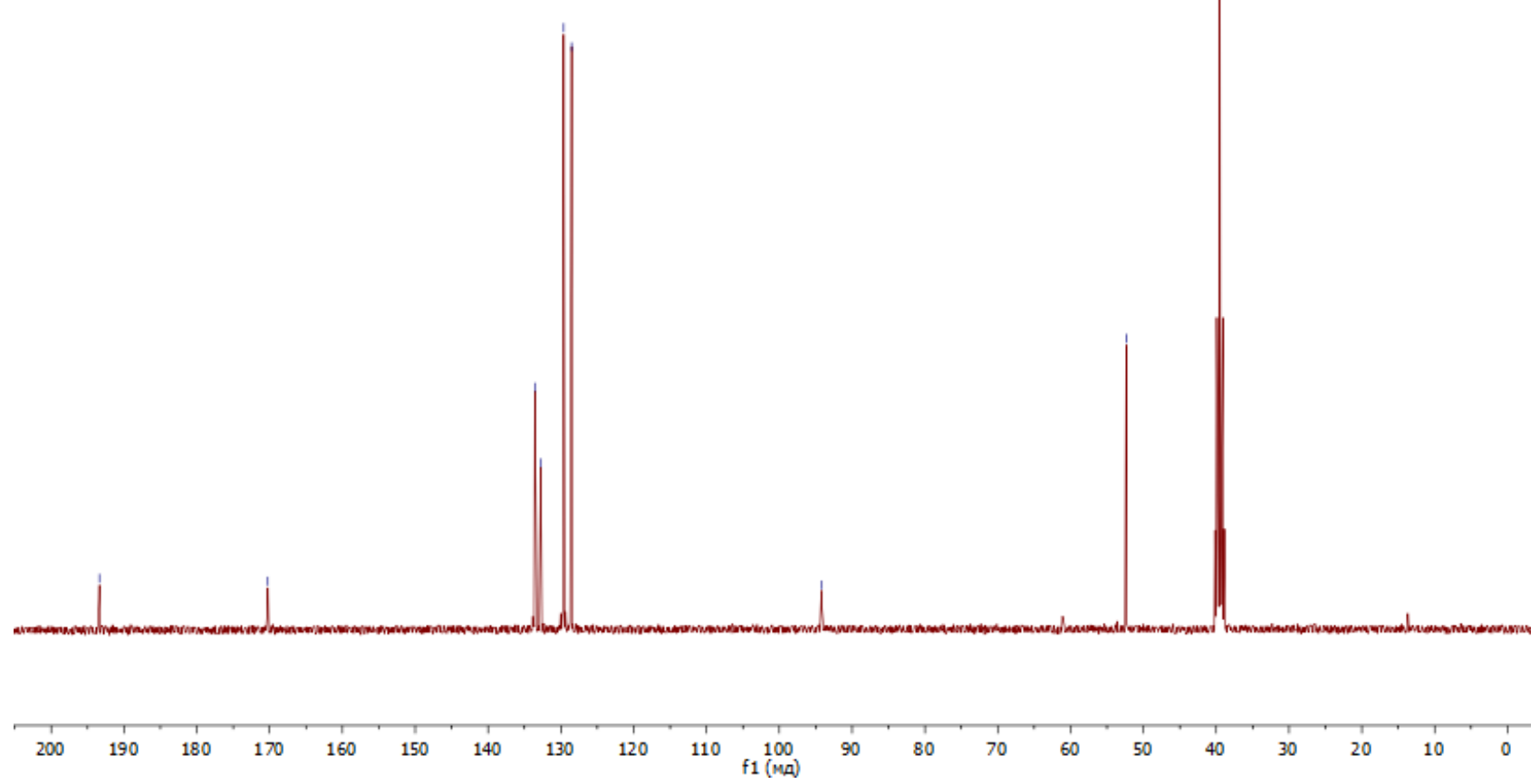

15 


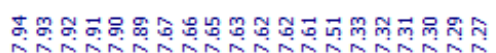

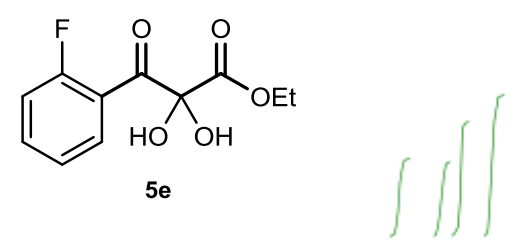

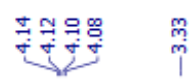

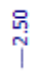

望

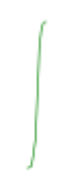

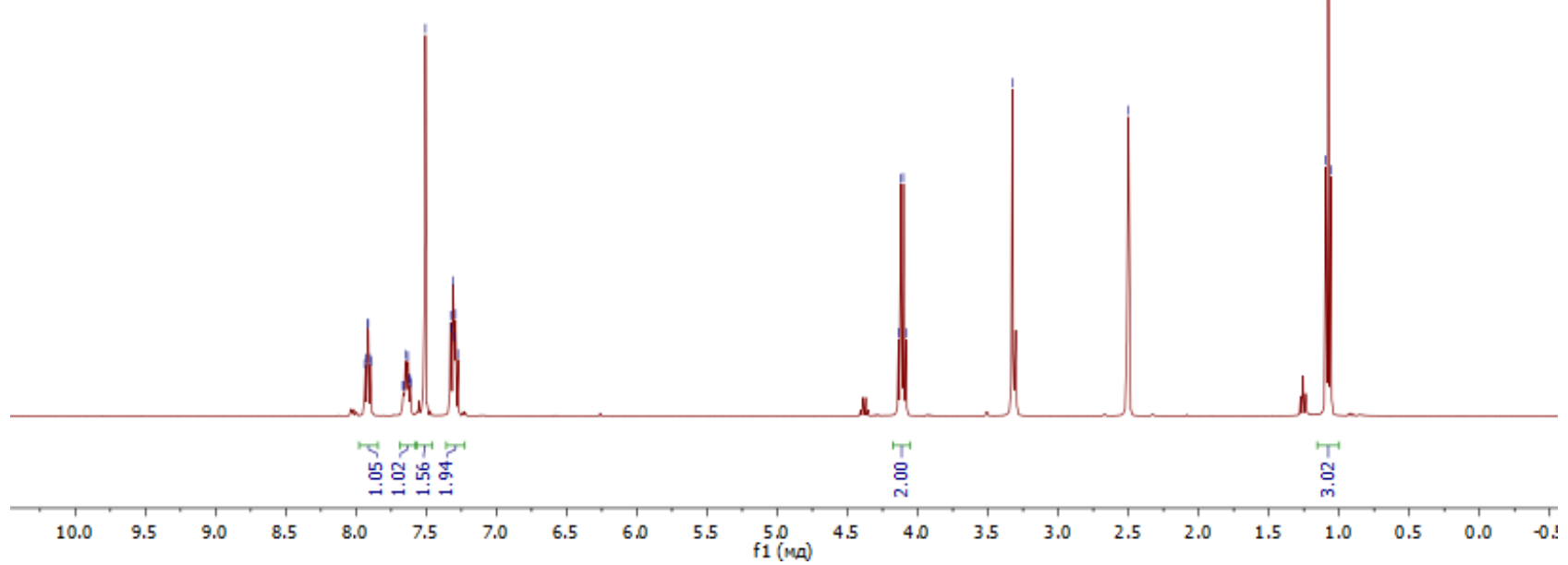

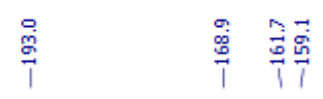

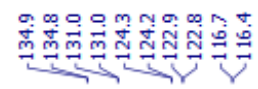

高

审

$\stackrel{m}{1}$

$\underbrace{\text { O }}_{\text {HO }}$

$5 e$


16 


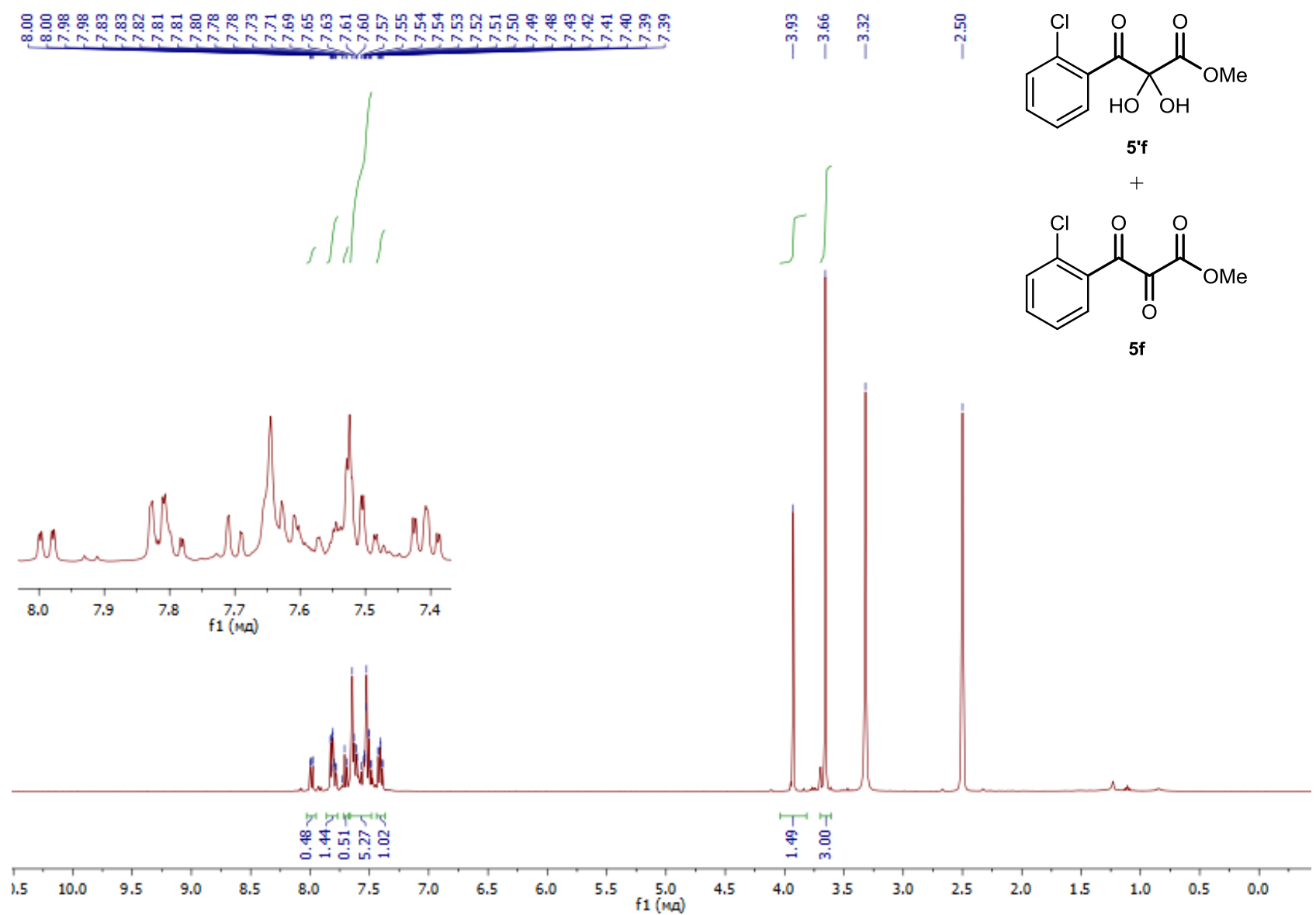

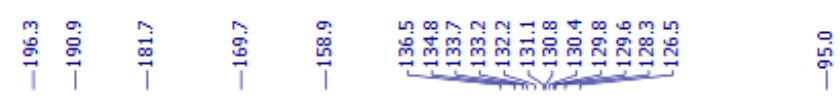
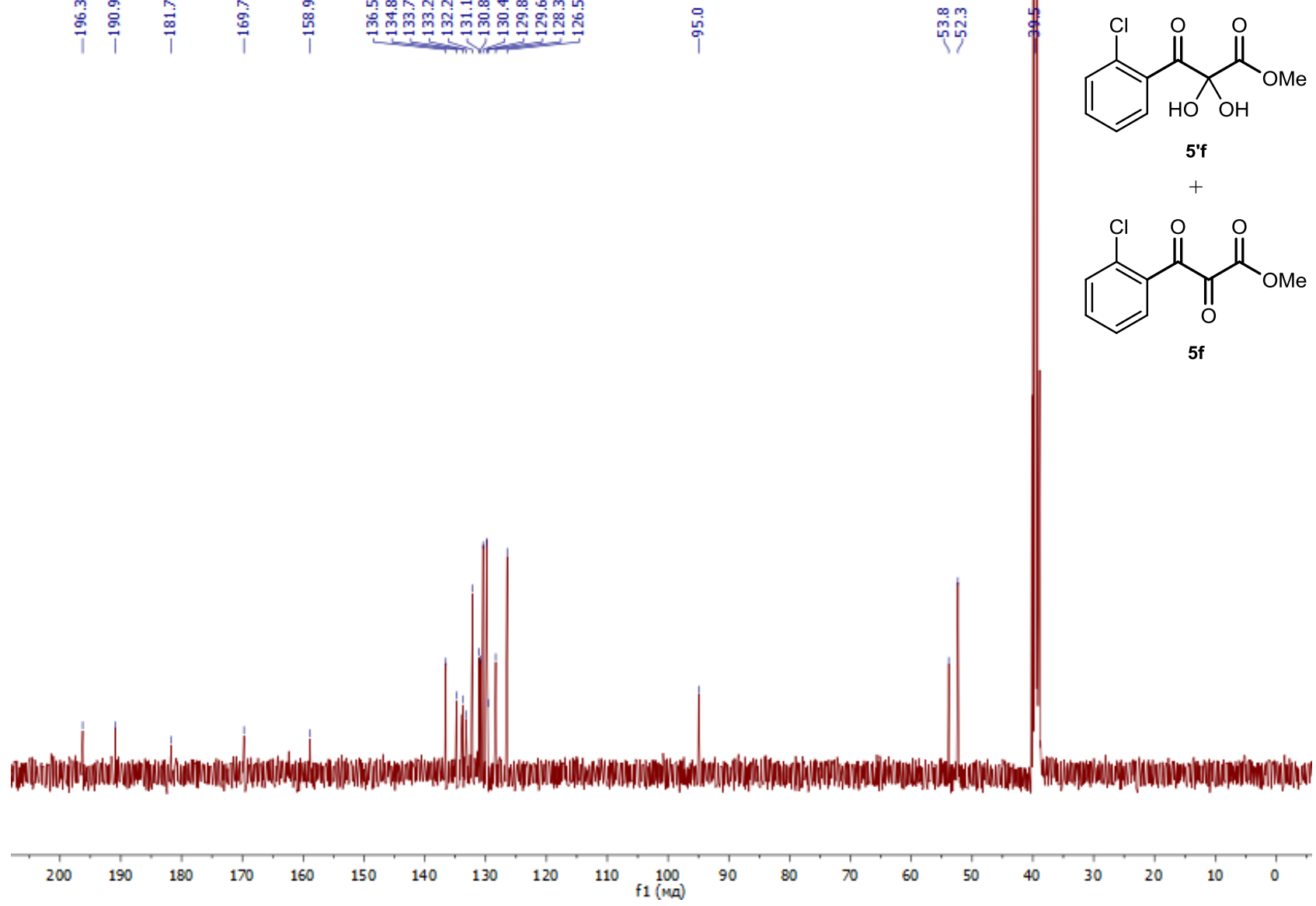

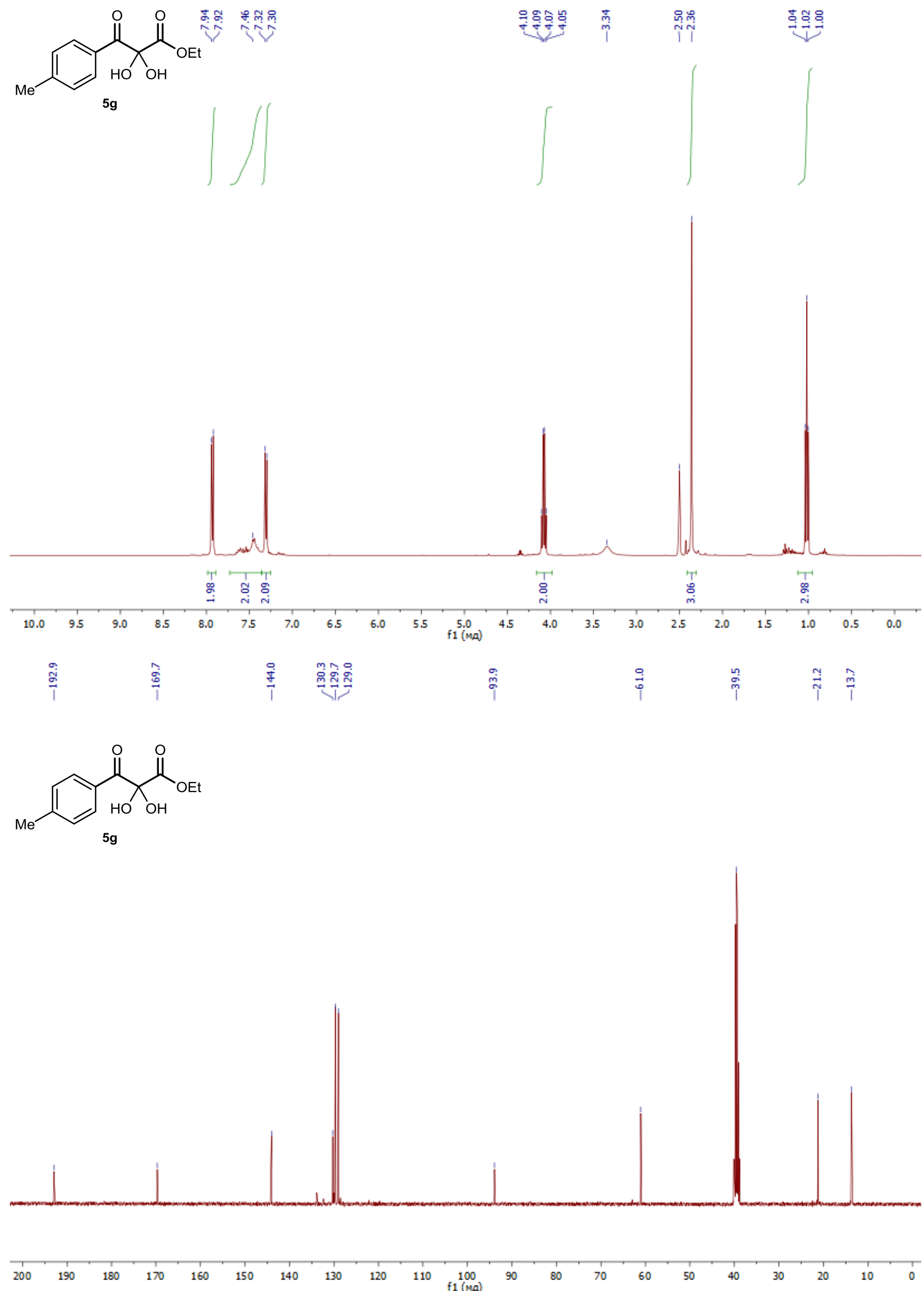


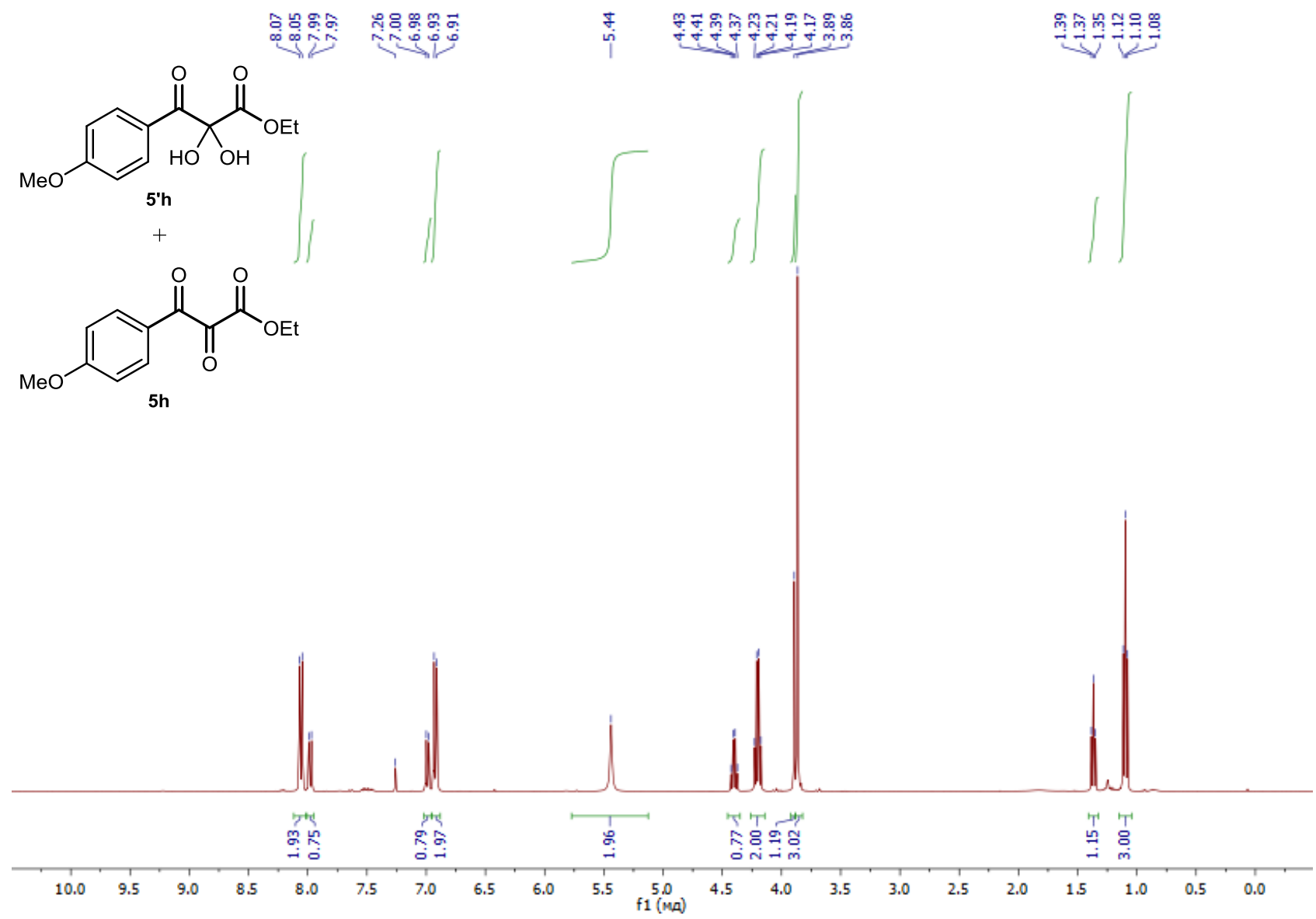

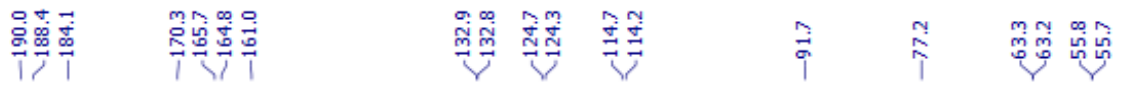

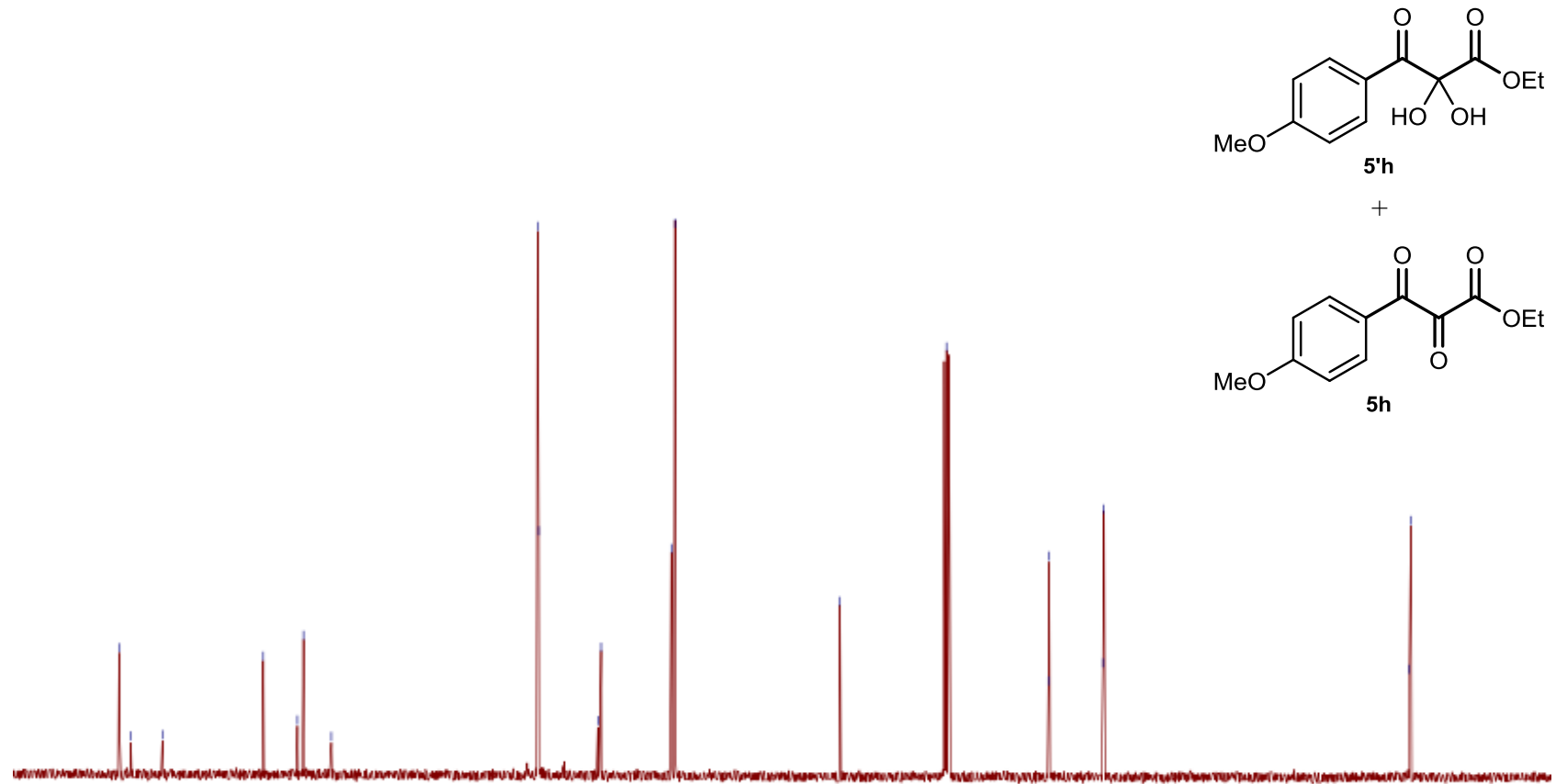

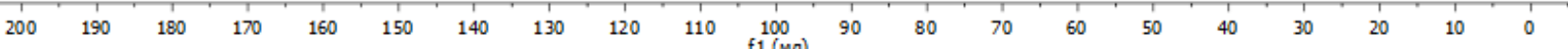




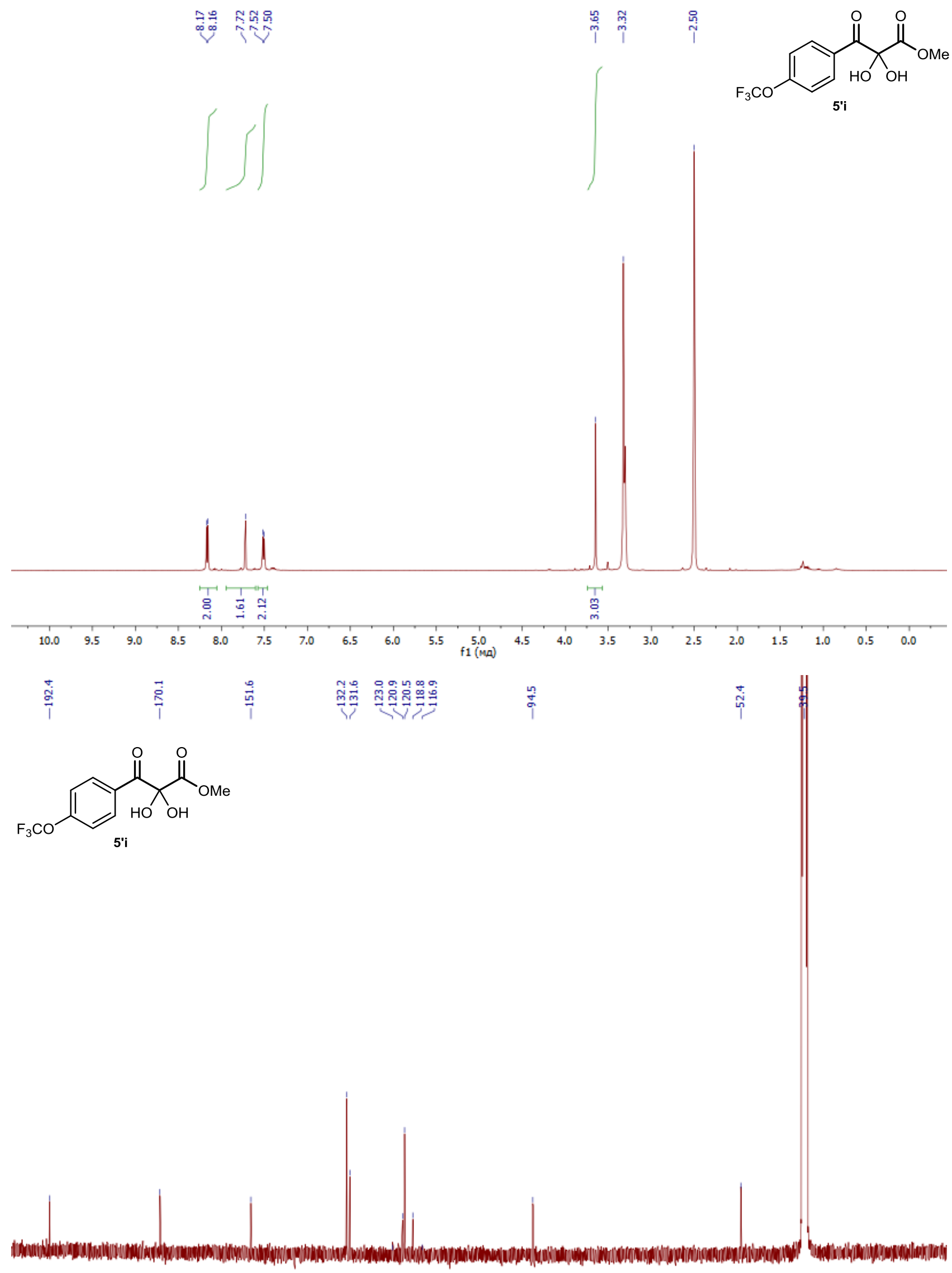

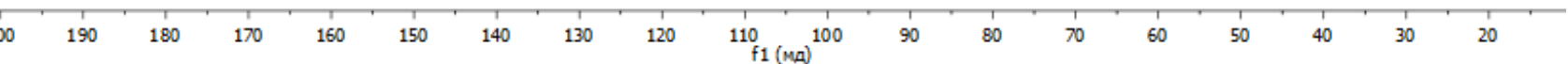




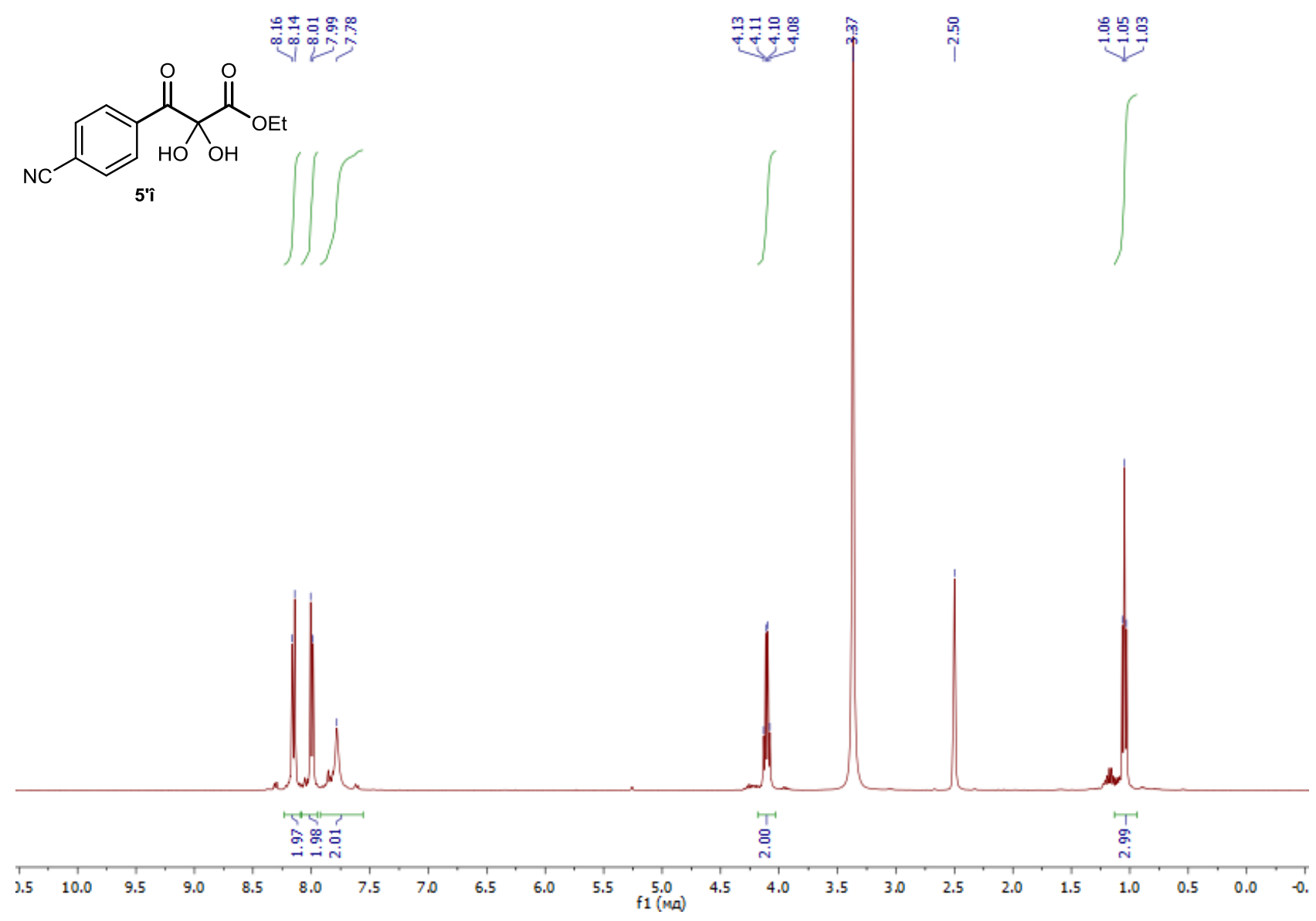

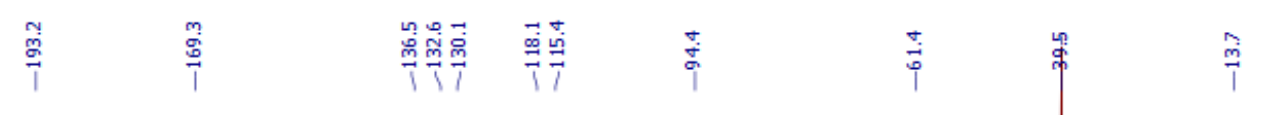<smiles>CCOC(=O)C(O)(O)C(=O)c1ccc(C#N)cc1</smiles>

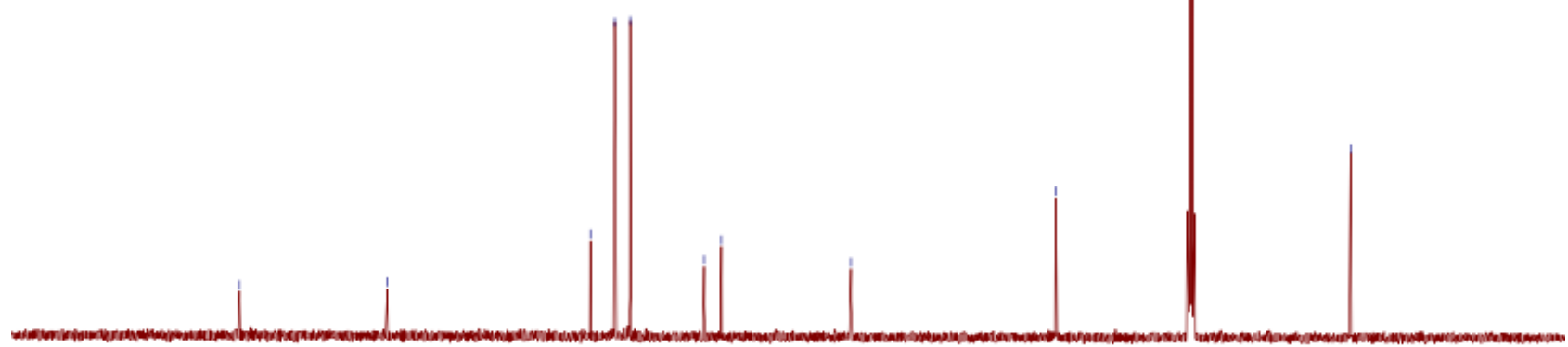

$\begin{array}{llllllllllllllllllllllllllll}10 & 220 & 210 & 200 & 190 & 180 & 170 & 160 & 150 & 140 & 130 & 120 & 110 & 100 & 90 & 80 & 70 & 60 & 50 & 40 & 30 & 20 & 10 & 0 & -10 & -21\end{array}$ 


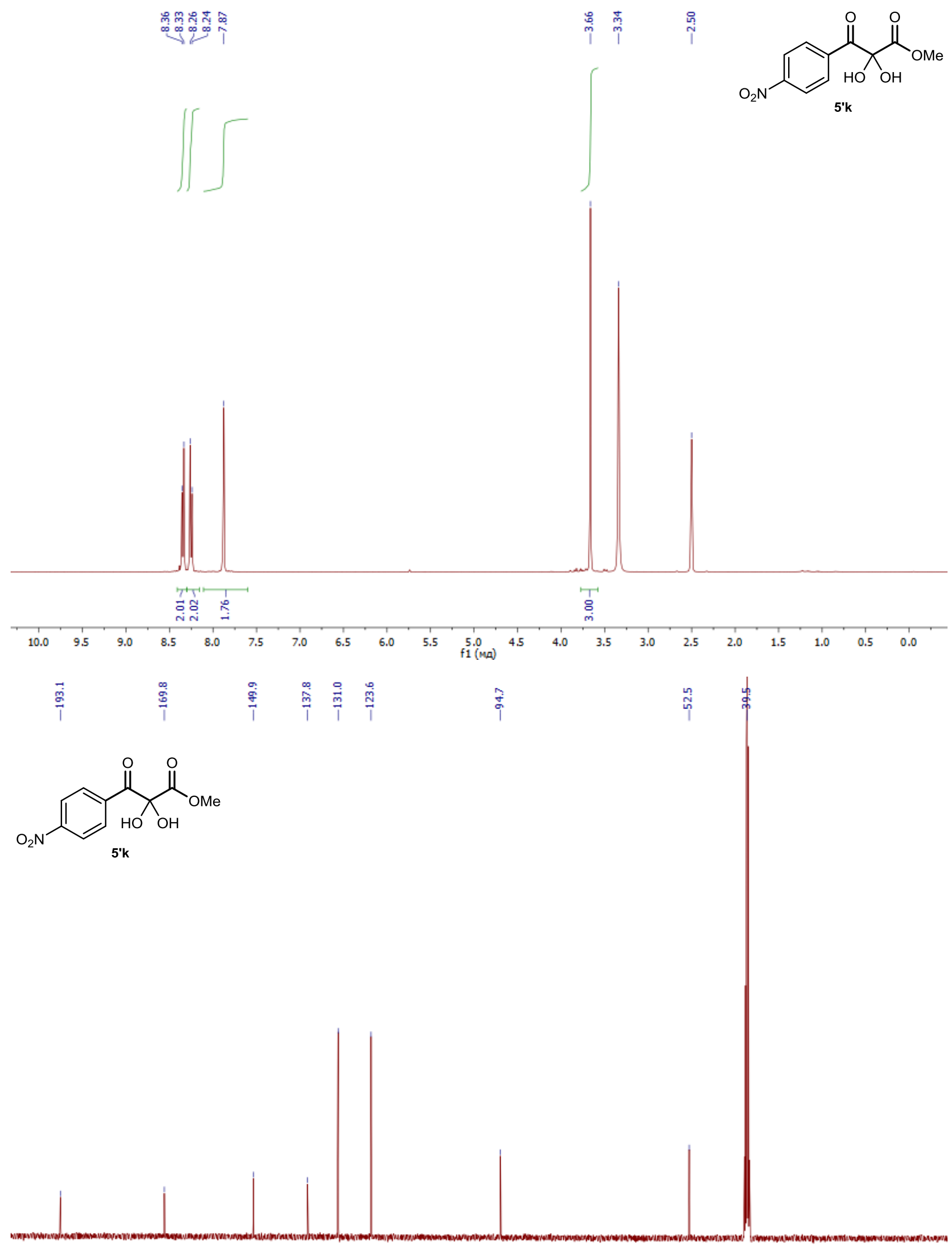

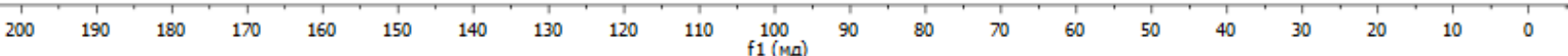




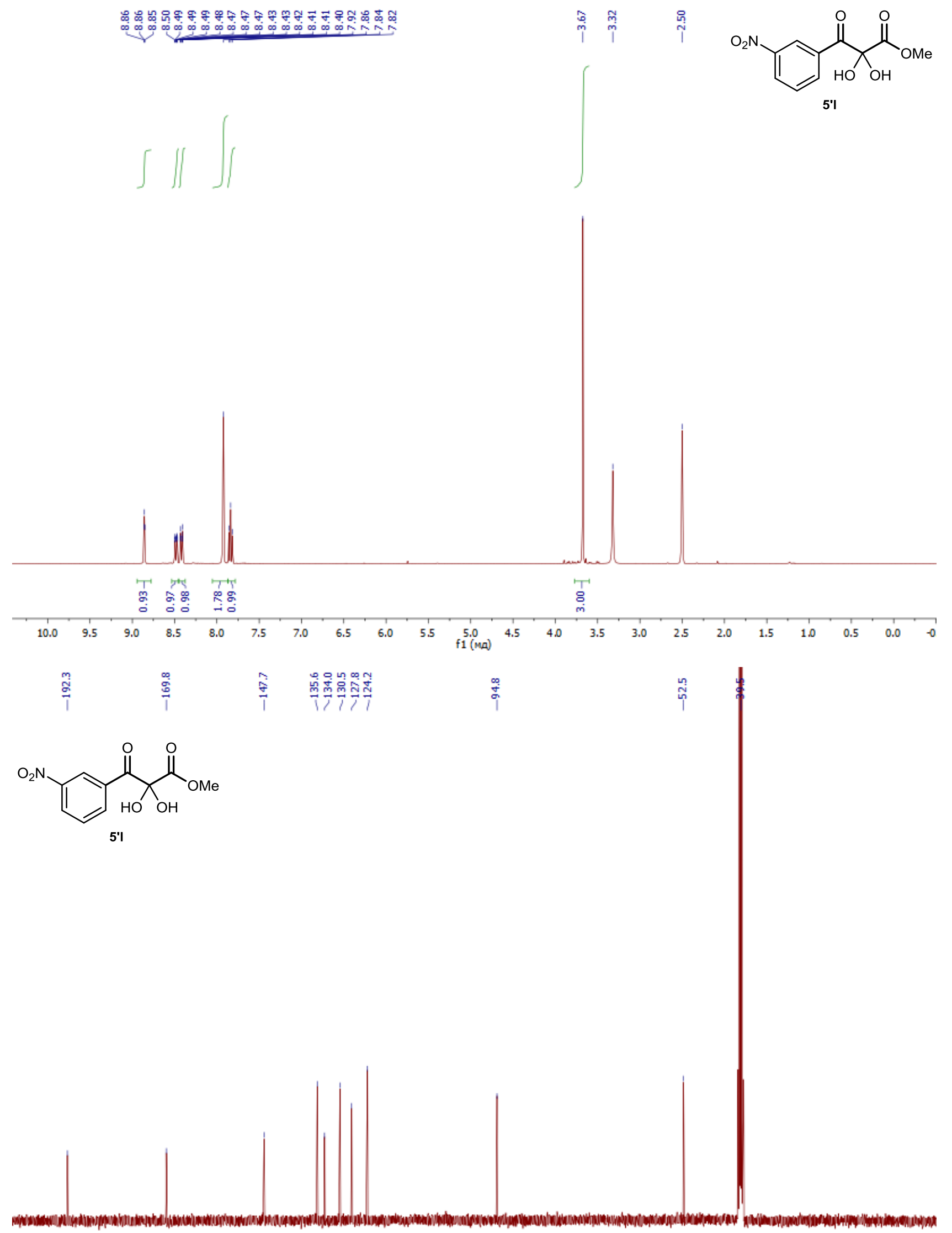

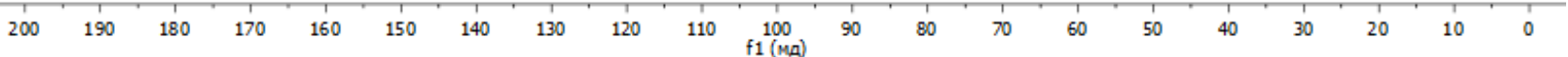




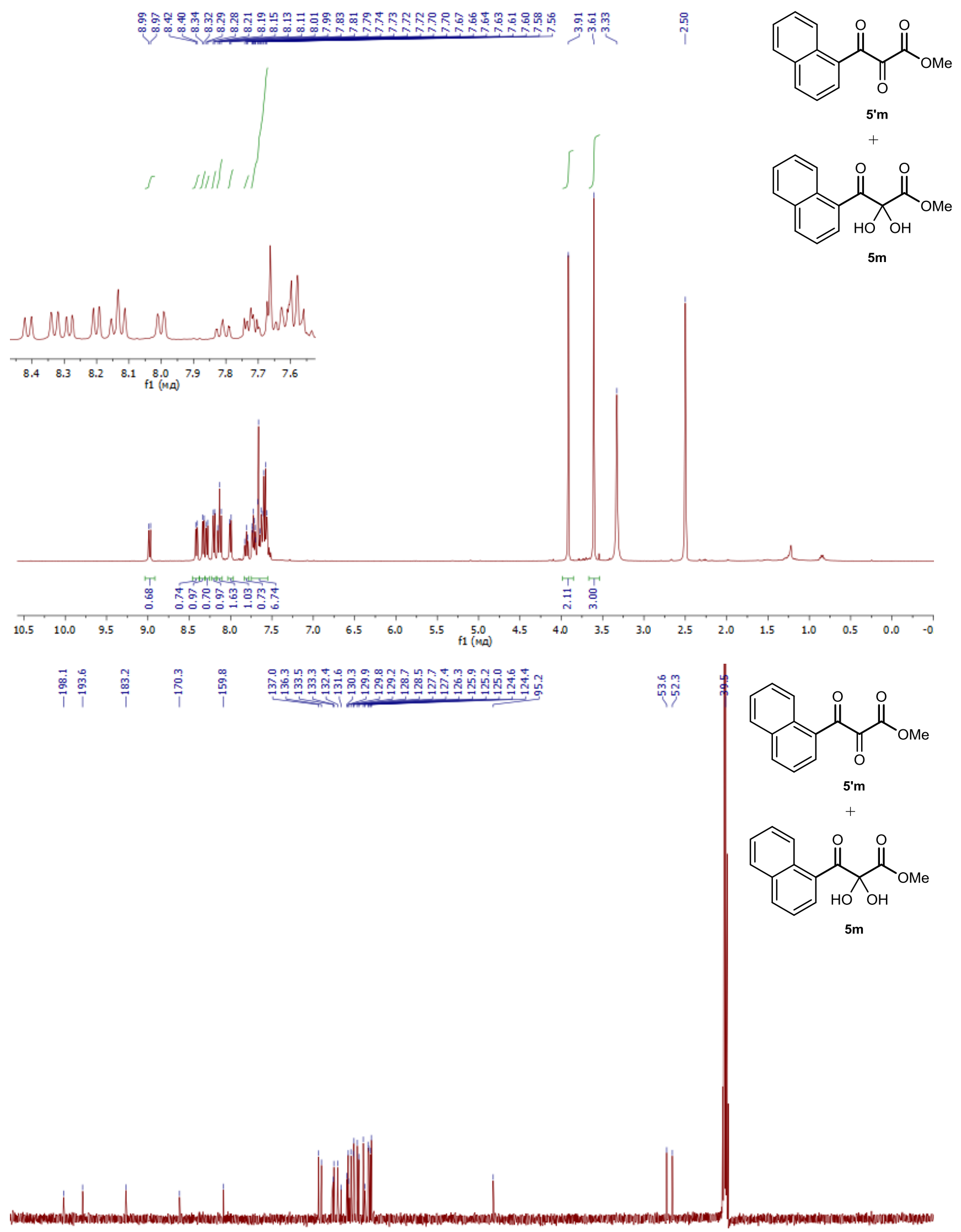

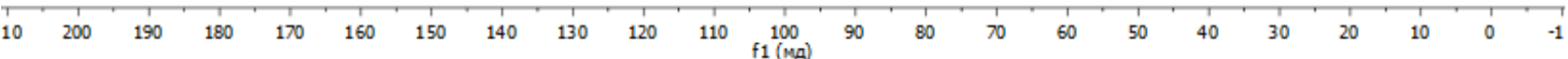


<smiles>CCOC(=O)C(O)(O)C(=O)c1cccs1</smiles>

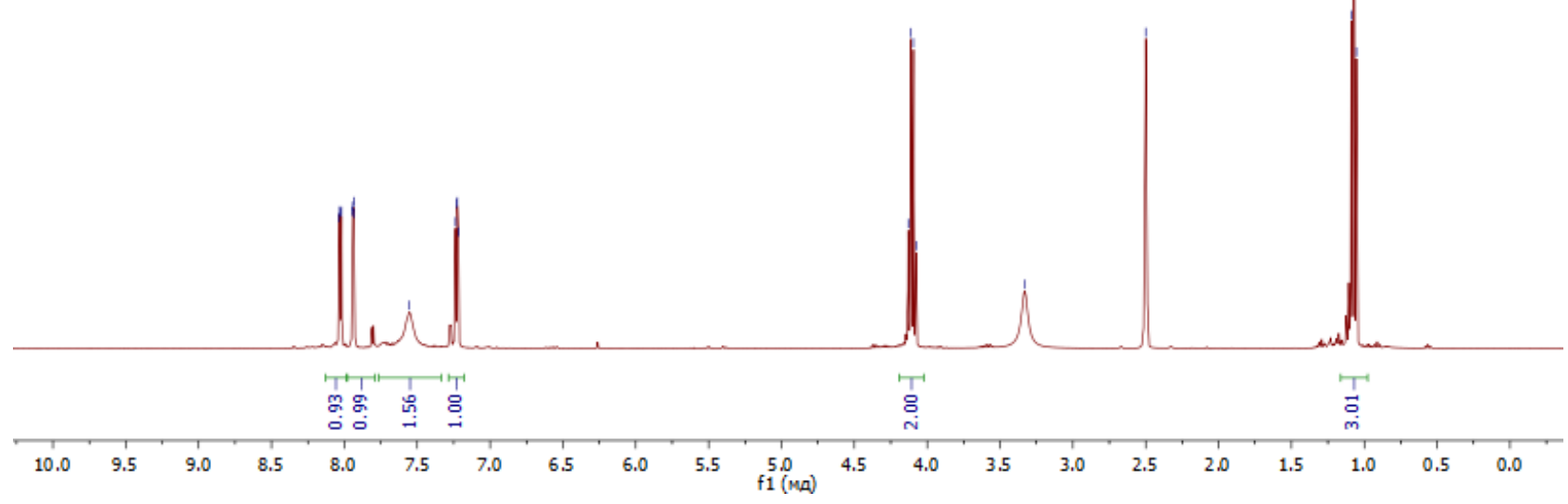

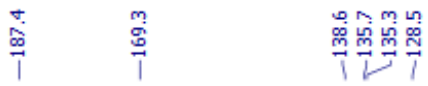

蓄<smiles>CCOC(=O)C(O)(O)C(=O)c1cccs1</smiles>

5'n

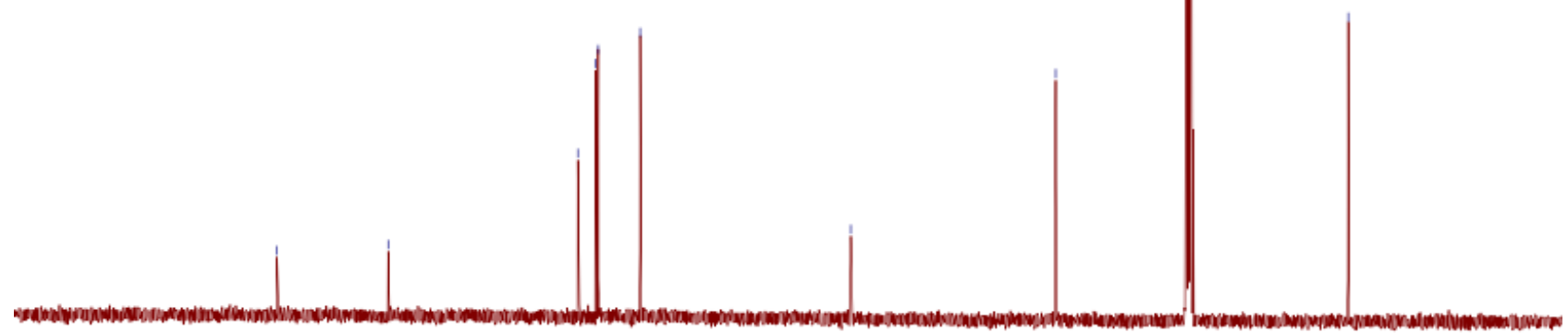

$\begin{array}{llllllllllllllllllllllllllll}10 & 220 & 210 & 200 & 190 & 180 & 170 & 160 & 150 & 140 & 130 & 120 & 110 & 100 & 90 & 80 & 70 & 60 & 50 & 40 & 30 & 20 & 10 & 0 & -10 & -21\end{array}$ 

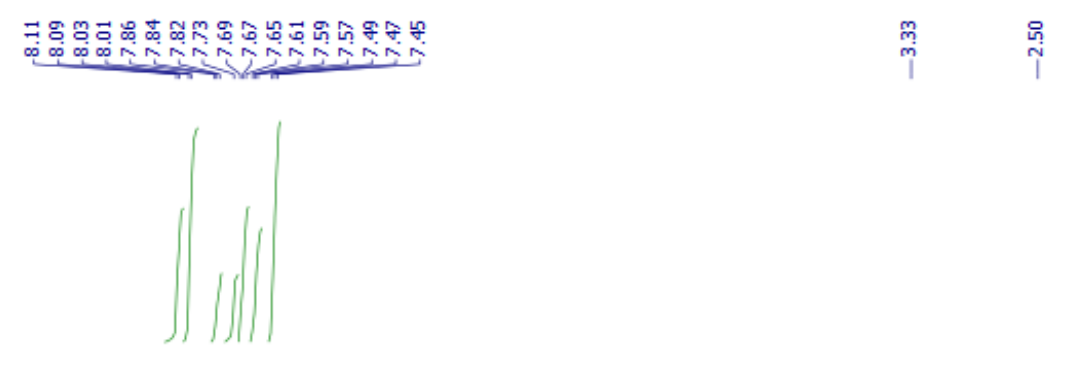

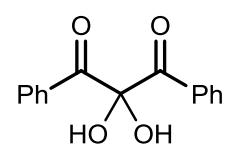

5'o

$+$<smiles>O=C(C(=O)c1ccccc1)C(=O)c1ccccc1</smiles>

50
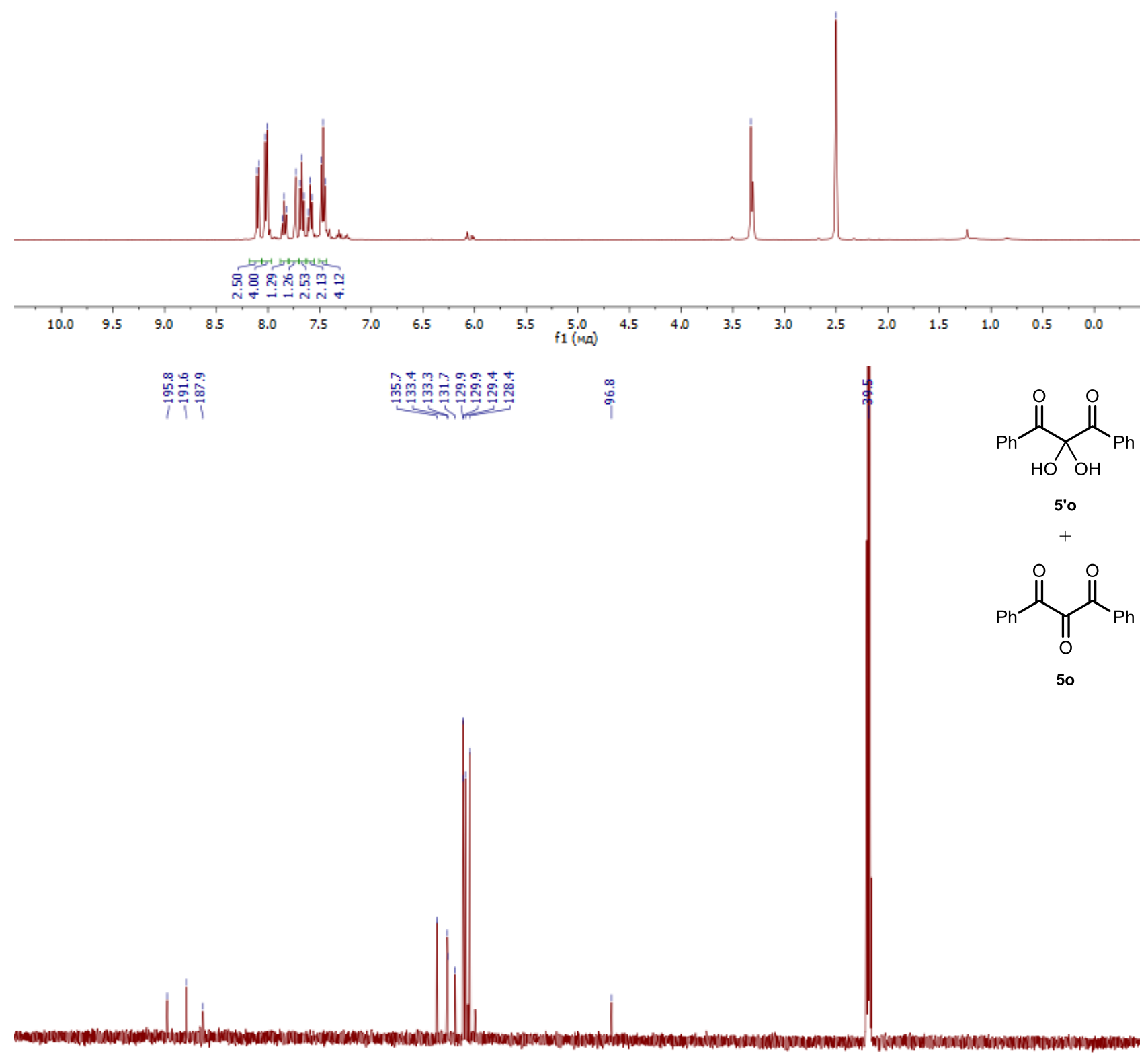

$220 \quad 210 \quad 200 \quad 190 \quad 180 \quad 170 \quad 160 \quad 150 \quad 140 \quad 130 \quad 120 \quad \begin{aligned} & 110 \\ & f 1(\mathrm{ma})\end{aligned}$ 


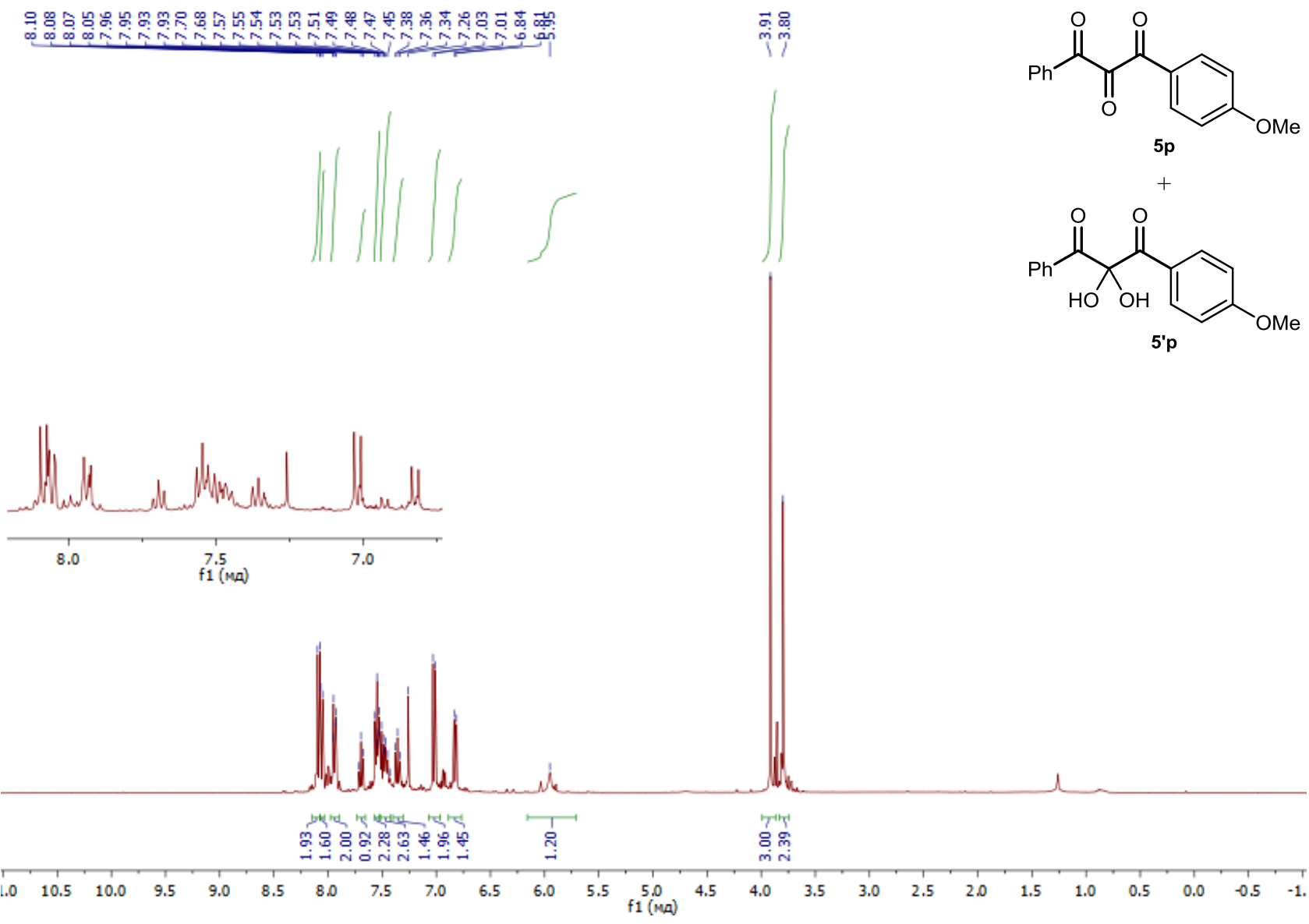

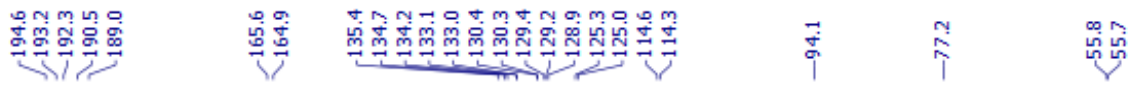

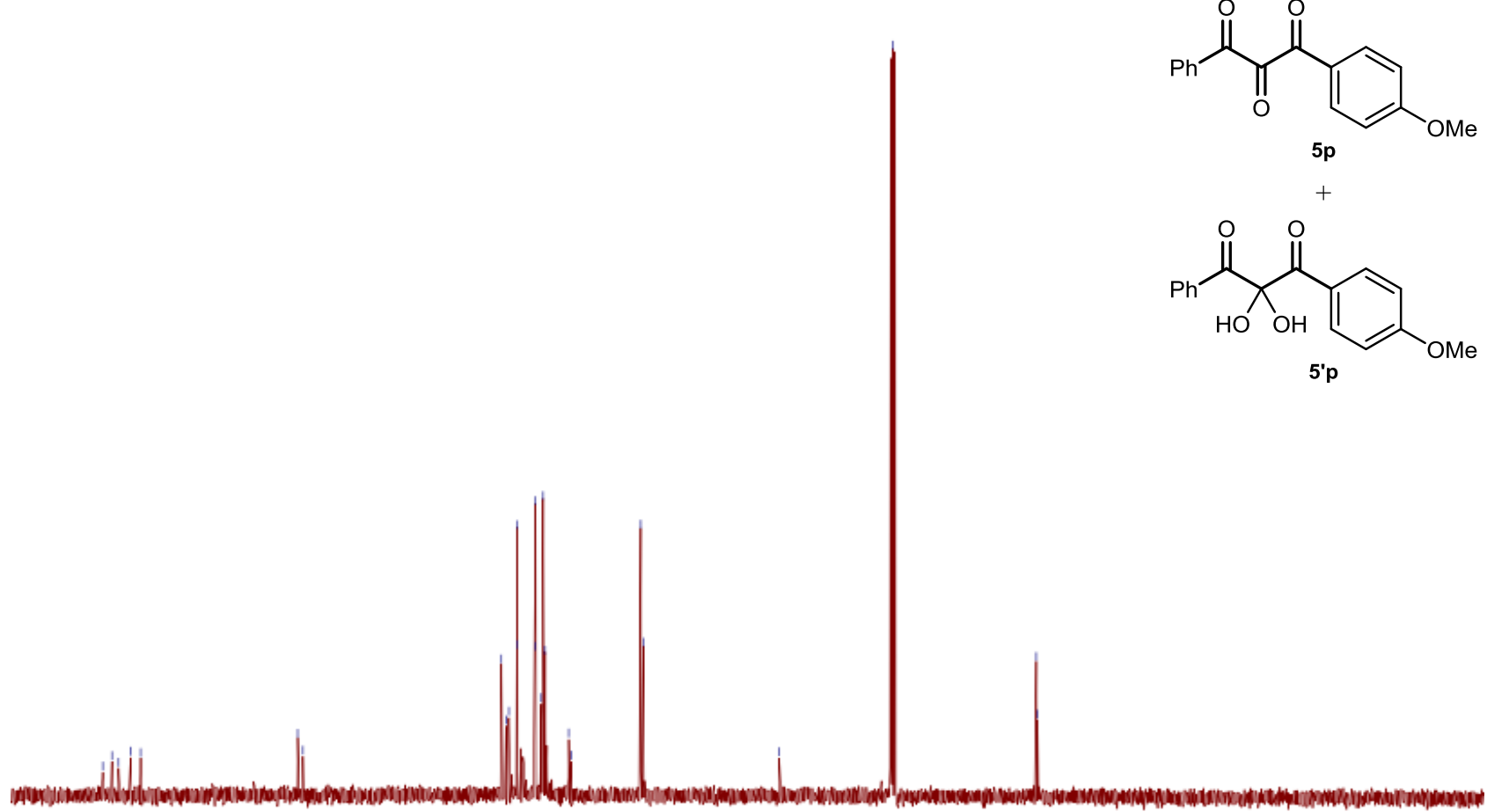

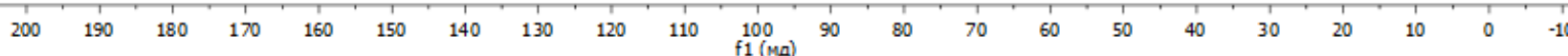


<smiles>Cc1ccccc1-c1ccccc1</smiles>

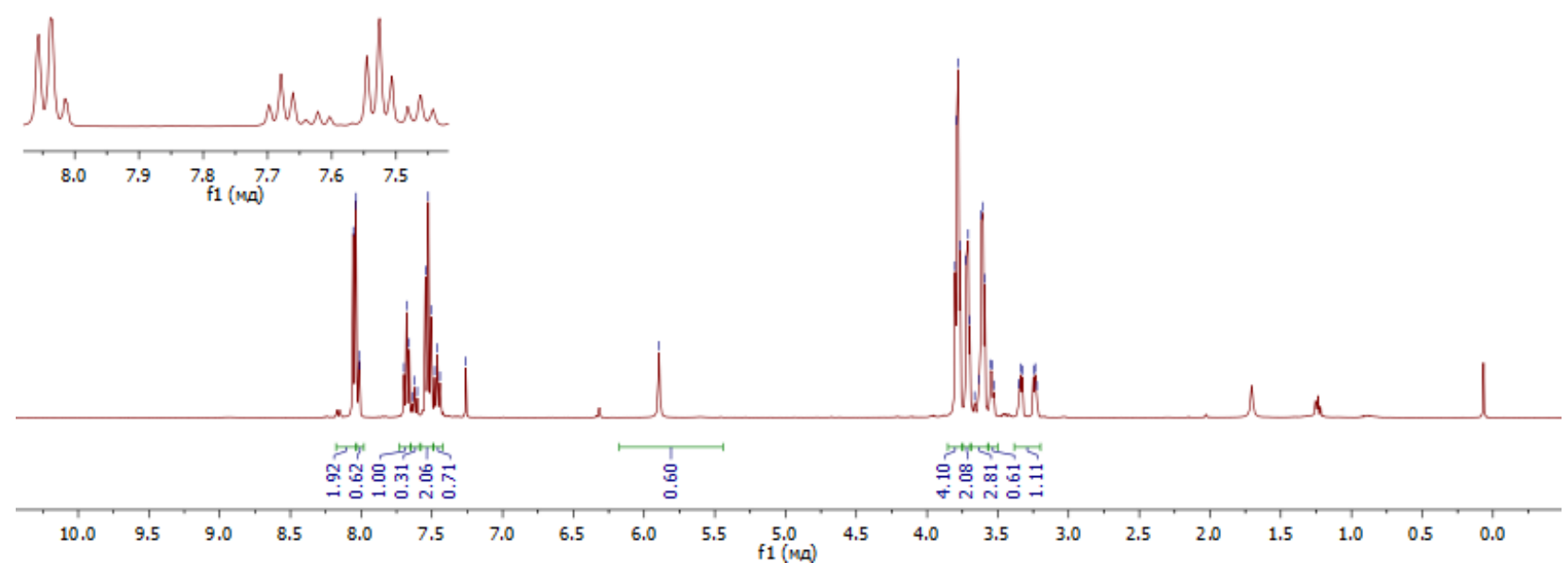

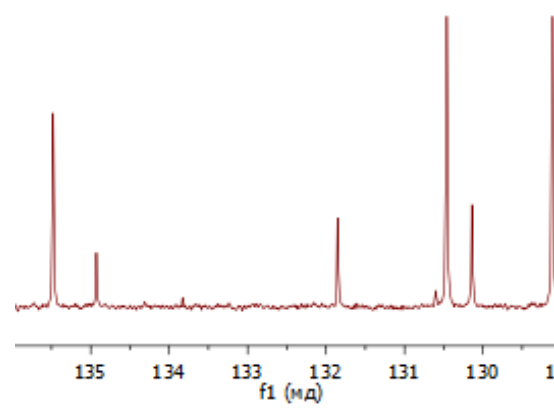

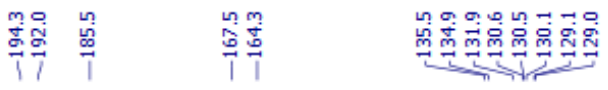

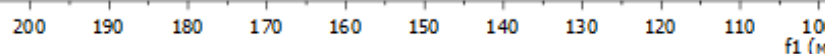<smiles>O=C(C(=O)c1ccccc1)C(=O)N1CCOCC1</smiles>

$5 '$ 


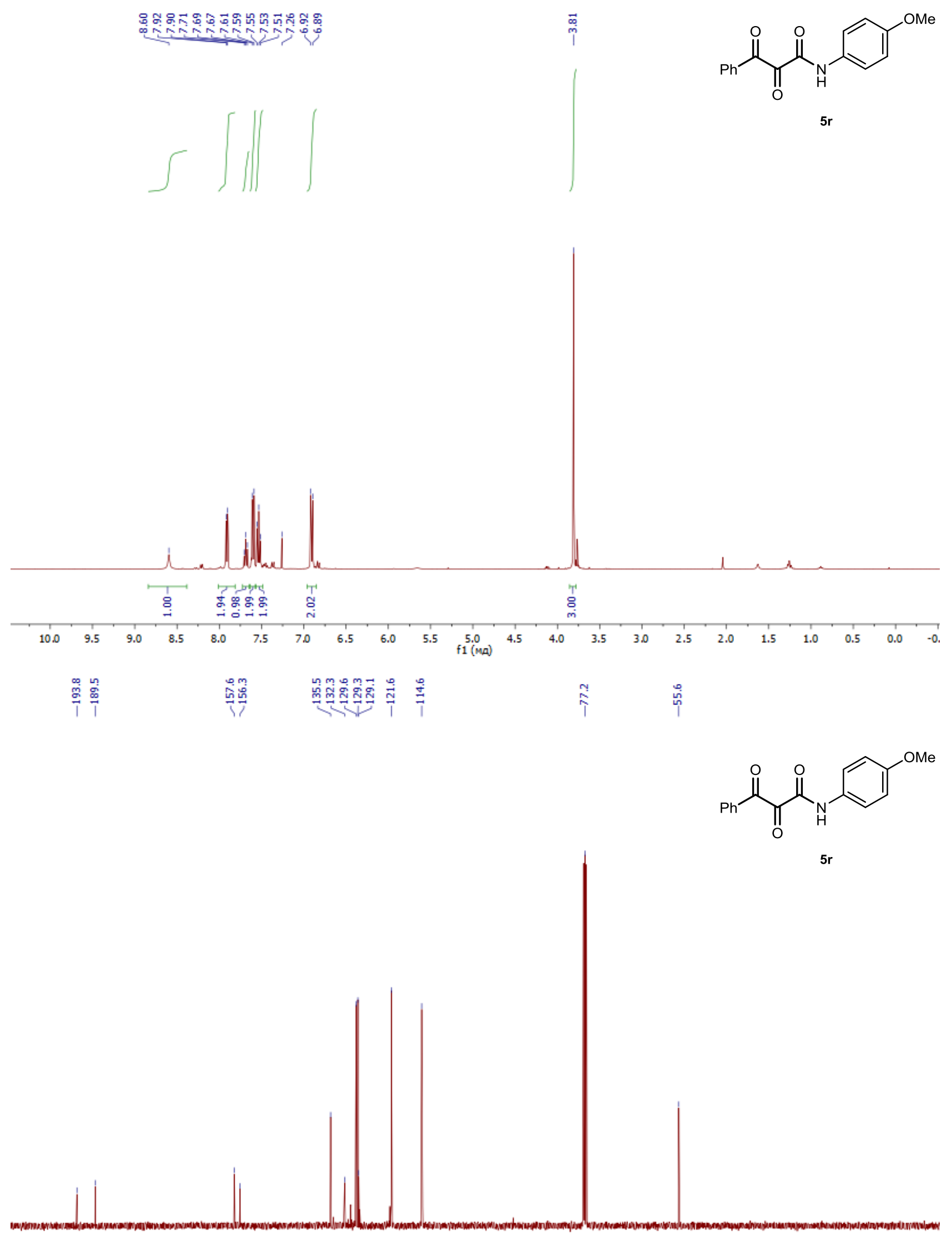

10.0

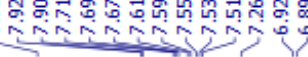

$\dddot{m}$

$\prod_{\mathrm{O}}$

$5 r$ 

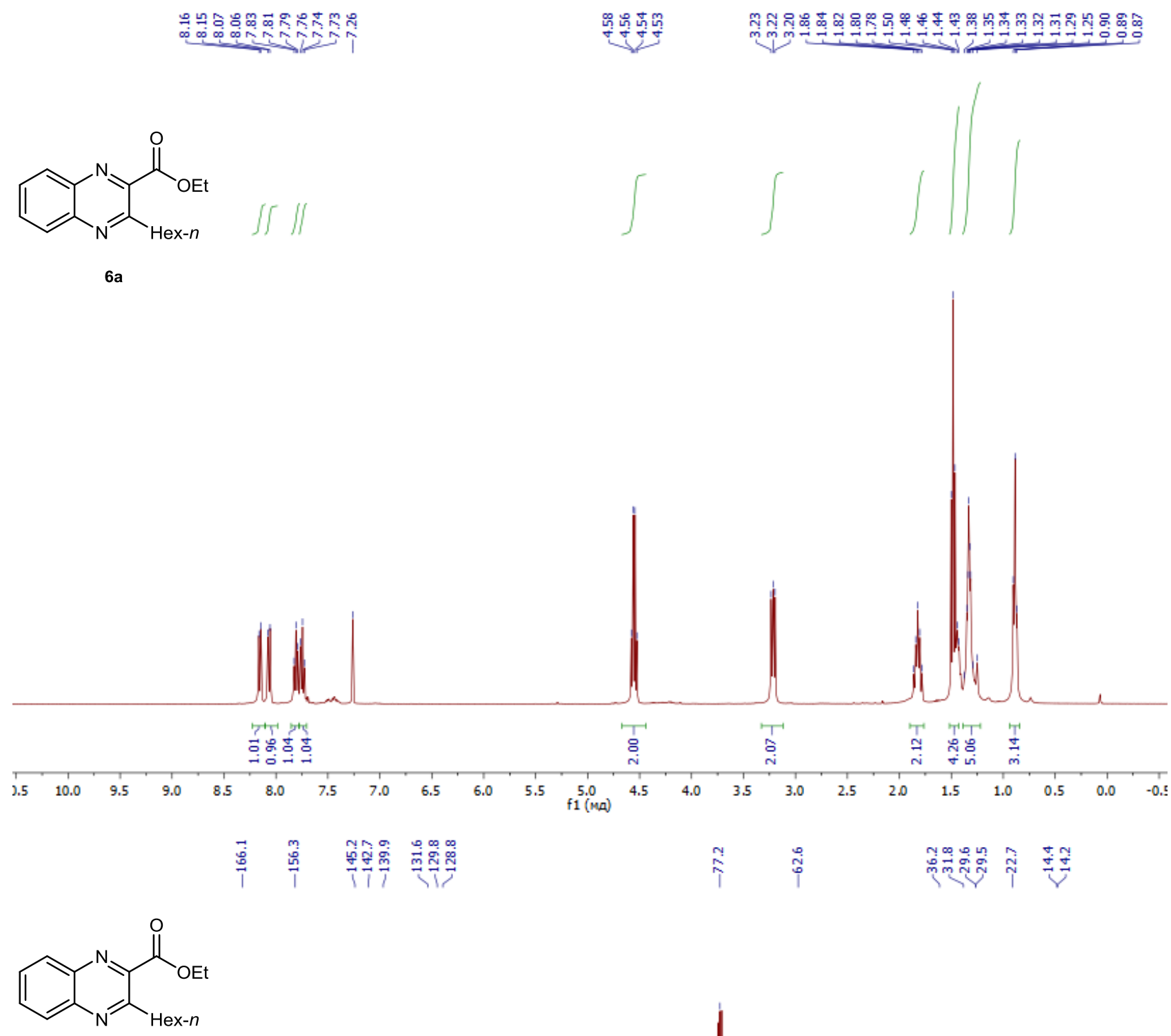

$6 a$

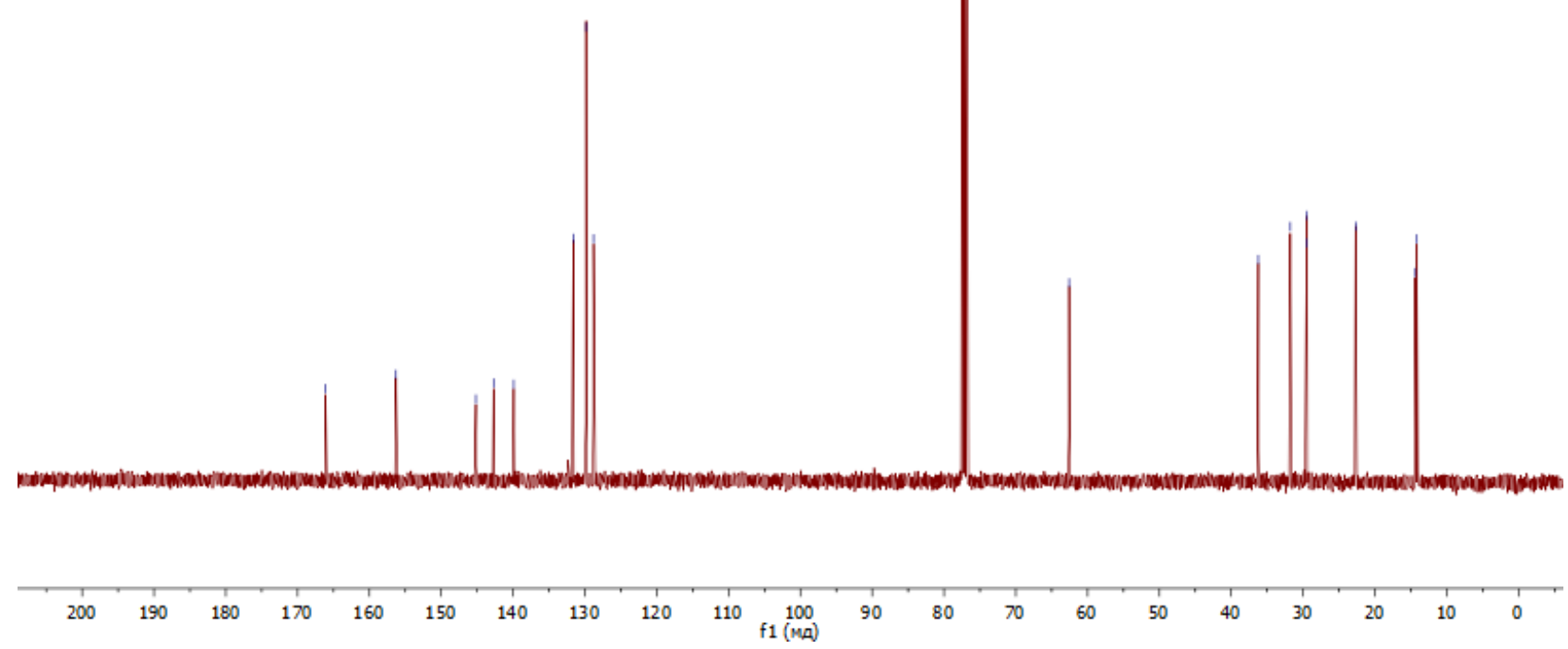




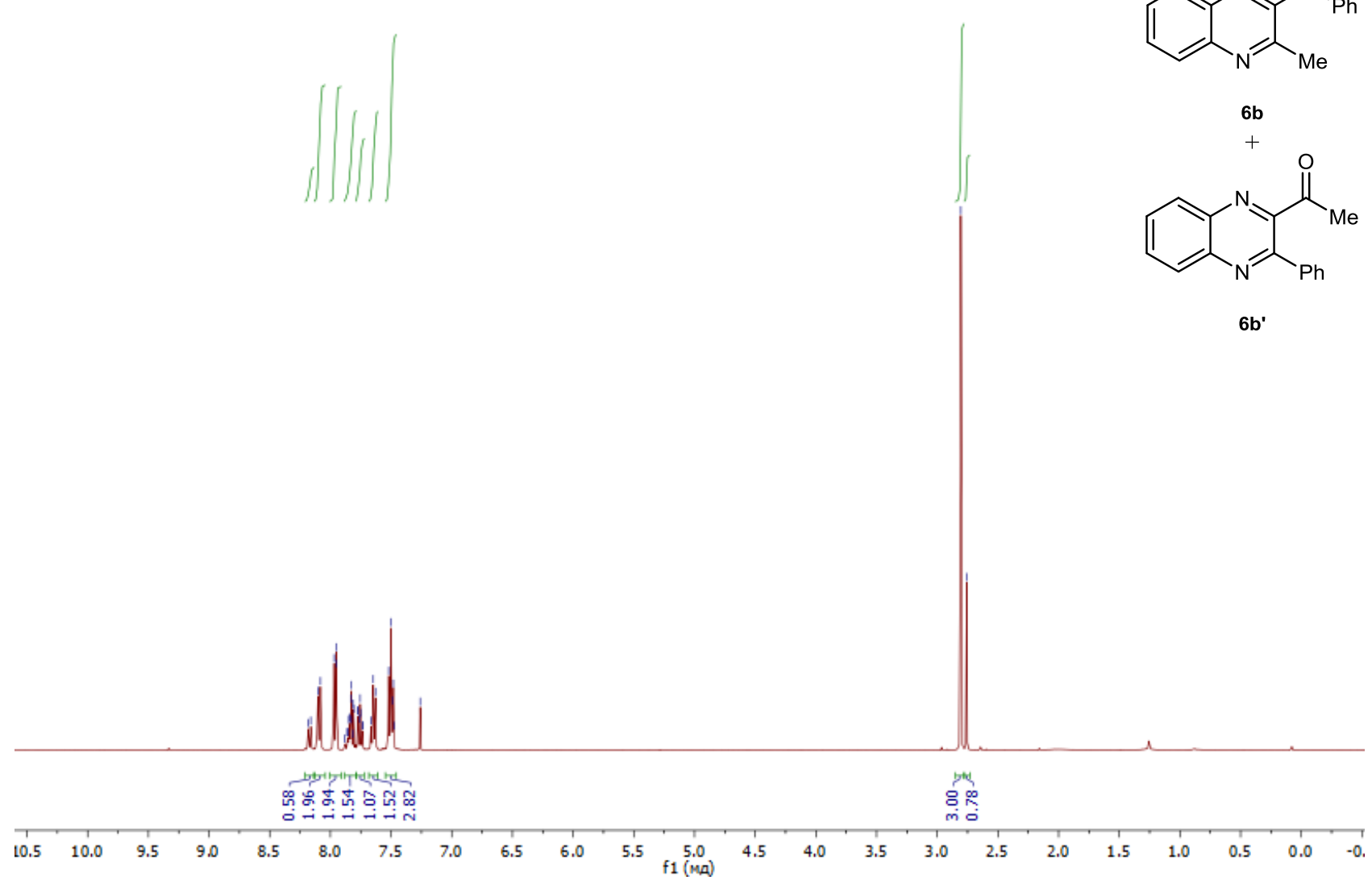

ণ্ণ

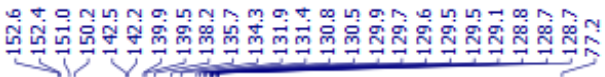

$\stackrel{\leftrightarrow}{\oplus} \stackrel{\sim}{\uparrow}$

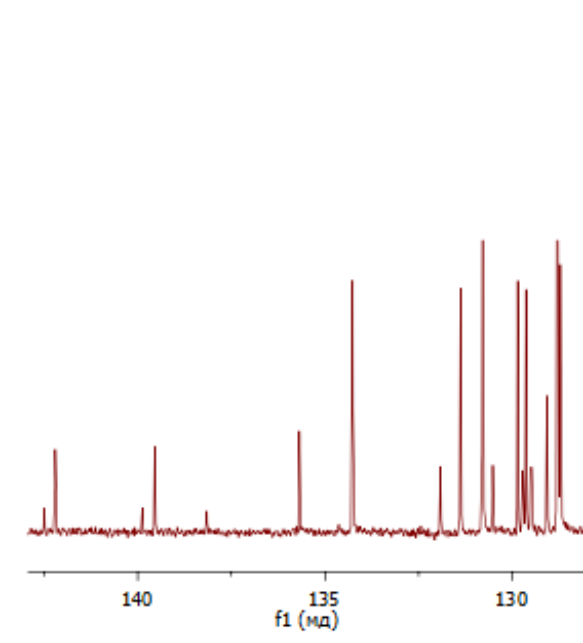<smiles>Cc1nc2ccccc2nc1C(=O)c1ccccc1</smiles>

6b

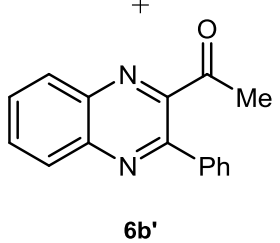

$\begin{array}{llllllllllllllllllllllllllllllllllllllll}30 & 220 & 210 & 200 & 190 & 180 & 170 & 160 & 150 & 140 & 130 & 120 & 110 & 100 & 90 & 80 & 70 & 60 & 50 & 40 & 30 & 20 & 10 & 0 & -10 & -2\end{array}$ 

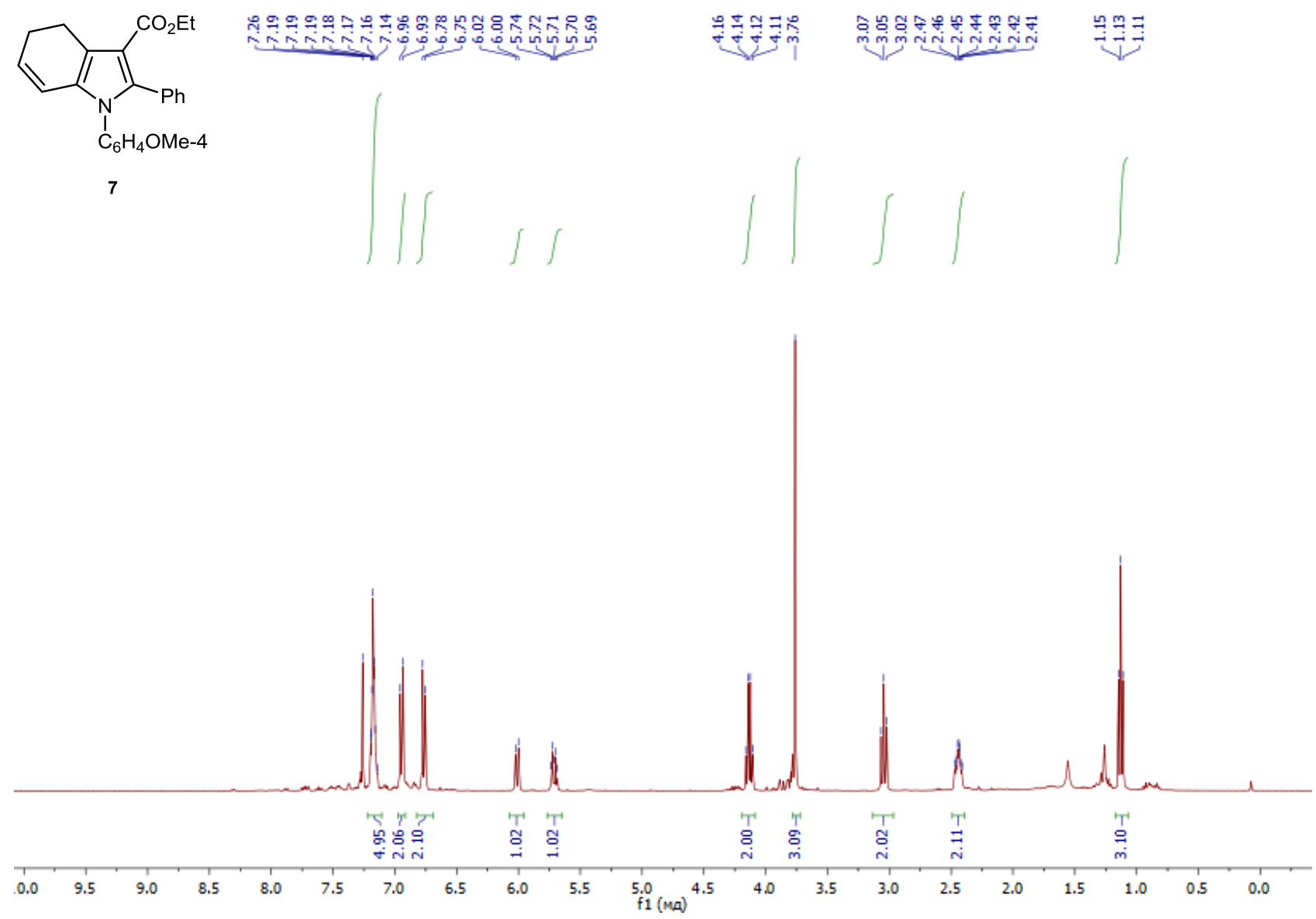

每

离 织

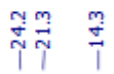

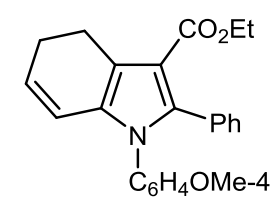

7

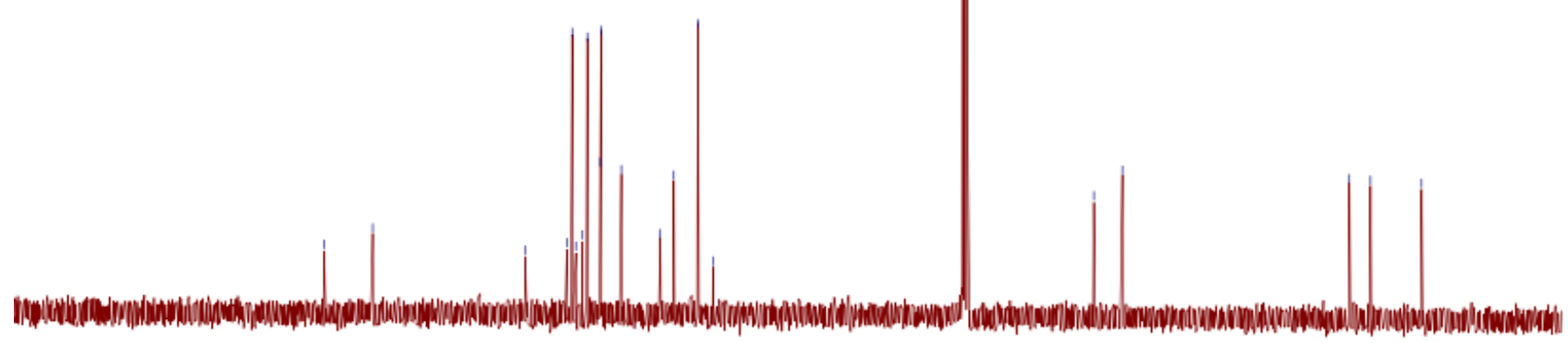



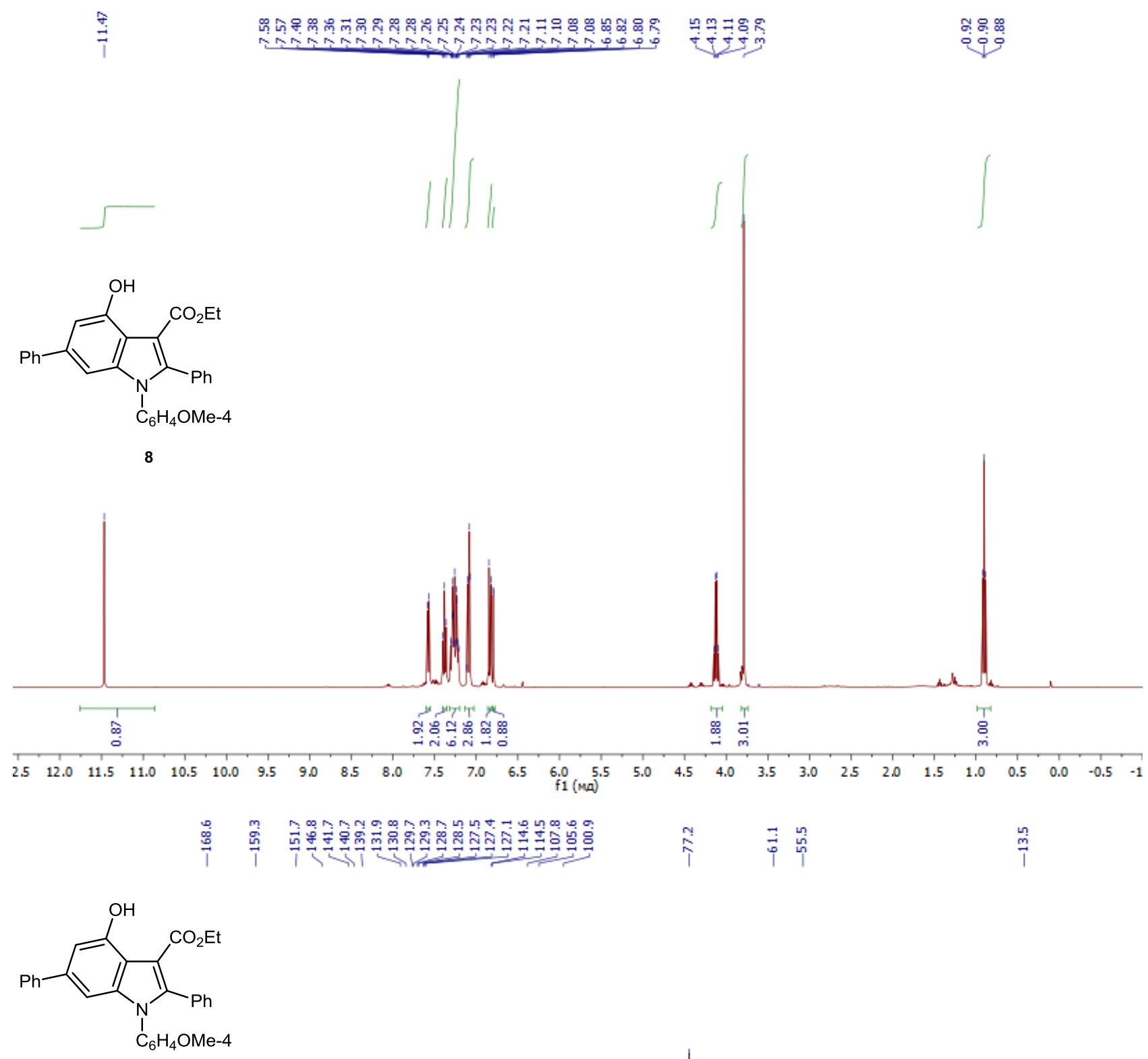

8

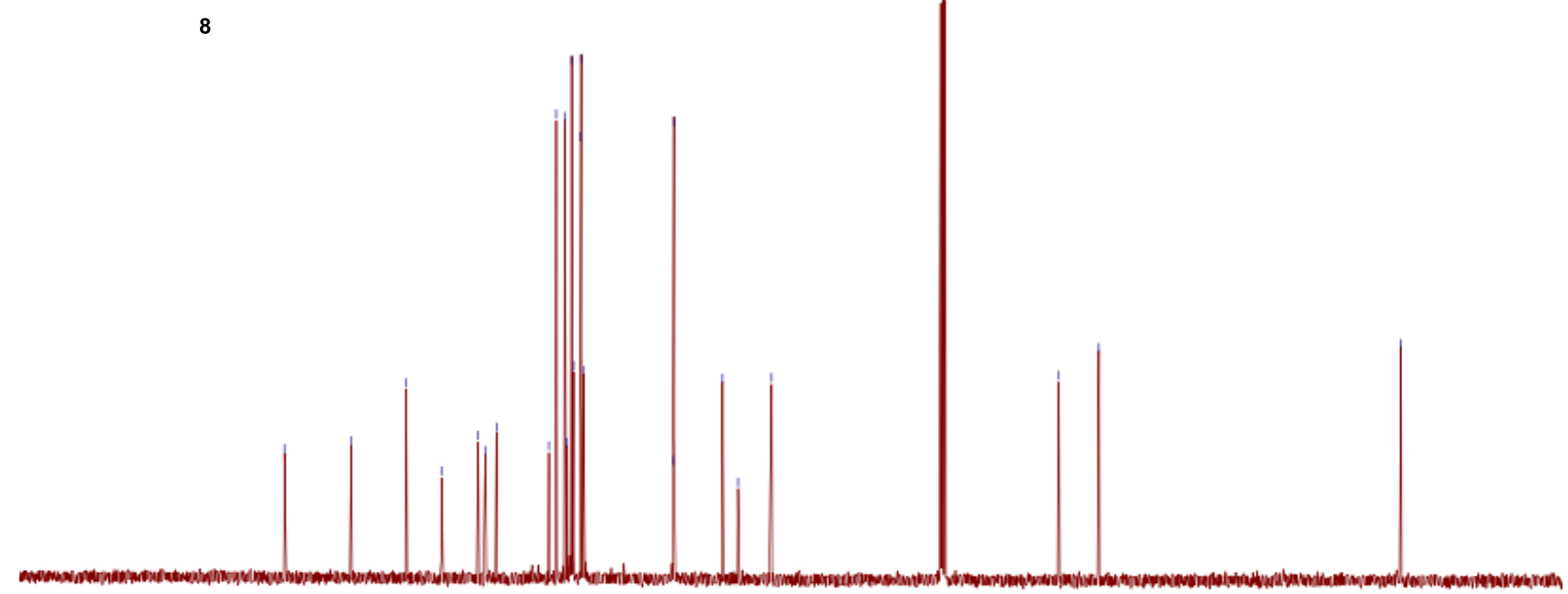

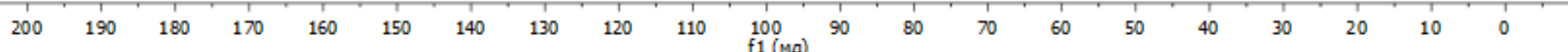



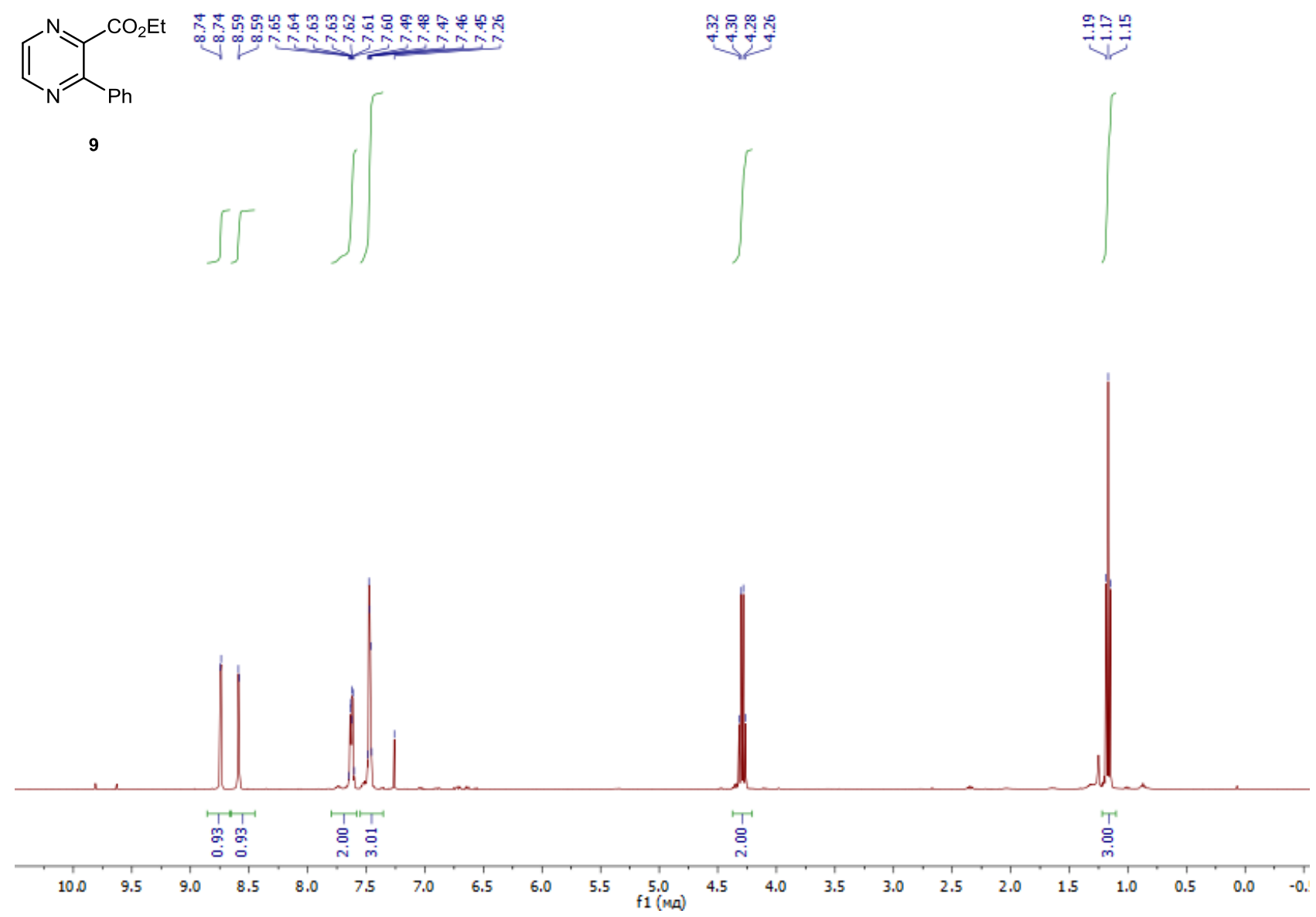

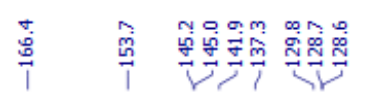

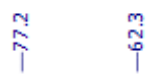

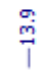<smiles>CCOC(=O)c1nccnc1-c1ccccc1</smiles>

9

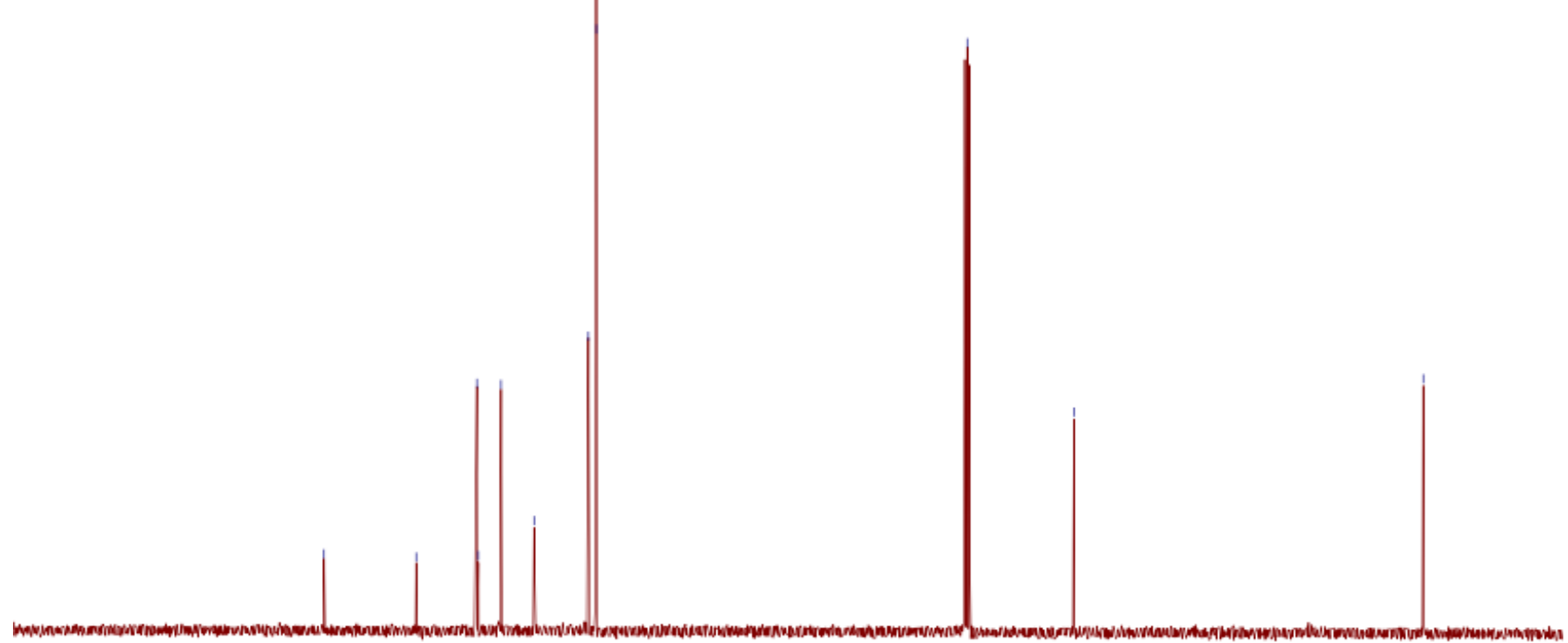

$\begin{array}{lllllllllll}200 & 190 & 180 & 170 & 160 & 150 & 140 & 130 & 120 & 110 & \begin{array}{l}100 \\ \mathrm{f} 1(\mathrm{ma})\end{array}\end{array}$ 
<smiles>CCOC(=O)C1(O)Oc2ccccc2N=C1c1ccccc1</smiles>
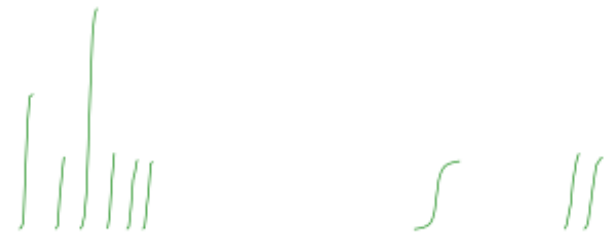

10

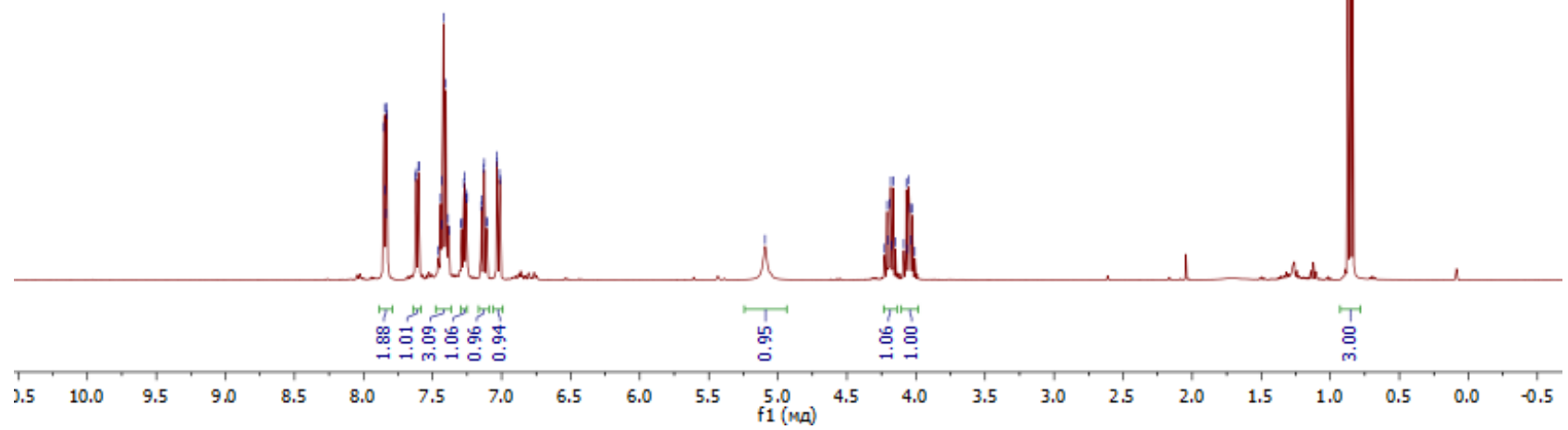

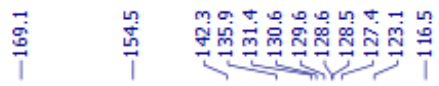

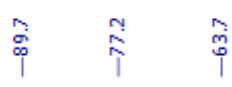<smiles>CCOC(=O)C1(O)Oc2ccccc2N=C1c1ccccc1</smiles>

10

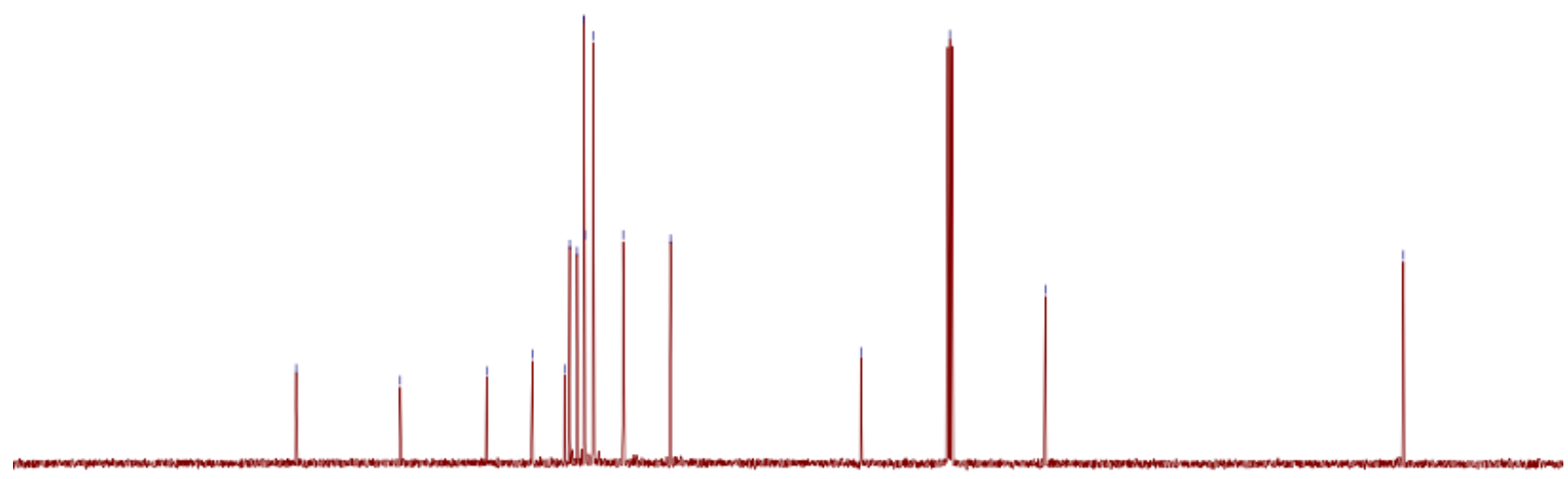

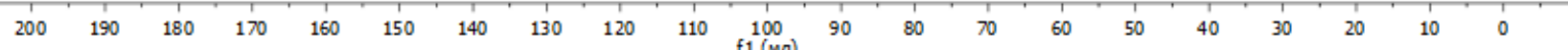

